\title{
Large Prandtl Number Asymptotics in Randomly Forced Turbulent Convection
}

\author{
Juraj Földes, Nathan Glatt-Holtz, Geordie Richards \\ emails: foldes@virginia.edu,negh@tulane.edu,geordie.richards@usu.edu
}

\begin{abstract}
We establish the convergence of statistically invariant states for the stochastic Boussinesq Equations in the infinite Prandtl number limit and in particular demonstrate the convergence of the Nusselt number (a measure of heat transport in the fluid). This is a singular parameter limit significant in mantle convection and for gasses under high pressure. The equations are subject to a both temperature gradient on the boundary and internal heating in the bulk driven by a stochastic, white in time, gaussian forcing. Here, the stochastic source terms have a strong physical motivation for example as a model of radiogenic heating.

Our approach uses mixing properties of the formal limit system to reduce the convergence of invariant states to an analysis of the finite time asymptotics of solutions and parameter-uniform moment bounds. Here, it is notable that there is a phase space mismatch between the finite Prandtl system and the limit equation, and we implement methods to lift both finite and infinite time convergence results to an extended phase space which includes velocity fields. For the infinite Prandtl stochastic Boussinesq equations, we show that the associated invariant measure is unique and that the dual Markovian dynamics are contractive in an appropriate Kantorovich-Wasserstein metric. We then address the convergence of solutions on finite time intervals, which is still a singular perturbation. In the process we derive wellposed equations which accurately approximate the dynamics up to the initial time when the Prandtl number is large.
\end{abstract}

Keywords: Convective Turbulence, Stochastic Boussinesq Equations, Large Prandtl Number Limit, Invariant Measures, Ergodicity, Kantorovich-Wasserstein Metrics, Singular Perturbation Analysis.

MSC2010: 76R05, 60H15, 35R60, 37L40

\section{Contents}

1 Introduction $\quad 2$

2 Mathematical Preliminaries and Main Results $\quad 5$

3 Reduction to Finite Time Dynamics $\quad 11$

4 Contraction in the Wasserstein Distance for the Infinite Prandtl System 12

5 Finite Time Asymptotics $\quad 17$

6 Convergence of the Nusselt number $\quad 25$

A Appendix: Moment Bounds for Stochastic Drift-Diffusion Equations 26

B Gradient Estimates on the Markov Semigroup 26

$\begin{array}{lr}\text { References } & 29\end{array}$ 


\section{Introduction}

Buoyancy driven convection plays a central role in a wide variety of physical processes: from earth's climate system to the internal dynamics of stars. As such it is of fundamental importance to identify and predict robust statistical quantities in these complex flows and to connect such statistics with the basic equations governing their dynamics, for example the Boussinesq equations. In particular characterizing pattern formation, mean heat transport, and small scale dynamics as a function of physical parameters and boundary conditions remains a topic of intensive research theoretically, numerically, and experimentally; see e.g. $[1,3,32,34]$ for a broad overview of recent developments.

It has long been understood that statistically invariant states of the nonlinear partial differential equations of fluid dynamics provide mathematical objects which are expected to contain various robust statistical quantities found in turbulent fluid flows. An ongoing challenge is therefore to address the existence, uniqueness, ergodicity, and dependence of these measures on parameters in a variety of specific contexts. While one may certainly pose such questions for deterministic equations (cf. [19]) the stochastic setting can be more tractable given the regularizing effect of noise on the associated probability distribution functions. Moreover, energy may be supplied to the system through both boundary or within the bulk of a fluid, the latter setting for instance models radioactive decay processes in the earth's mantle; see [2, 24, 33, 39, 41, 49]. Both sources can therefore have an essentially stochastic character in situations of physical interest.

In this and a companion work, [22], we study statistically invariant states of the stochastically driven Boussinesq equations

$$
\begin{gathered}
\frac{1}{P r}\left(\partial_{t} \mathbf{u}+\mathbf{u} \cdot \nabla \mathbf{u}\right)-\Delta \mathbf{u}=\nabla p+R a \hat{\mathbf{k}} T, \quad \nabla \cdot \mathbf{u}=0, \\
d T+\mathbf{u} \cdot \nabla T d t=\Delta T d t+\sum_{k=1}^{N} \sigma_{k} d W^{k},
\end{gathered}
$$

for the (non-dimensionalized) velocity field $\mathbf{u}=\left(u_{1}, u_{2}, u_{3}\right)$, pressure $p$, and temperature $T$ of a buoyancy driven fluid. The system (1.1)-(1.2) evolves in a three dimensional domain $(x, y, z)=(\mathbf{x}, z) \in \mathcal{D}=[0, L]^{2} \times$ $[0,1]$ and is supplemented with the boundary conditions

$$
\mathbf{u}_{\mid z=0}=\mathbf{u}_{\mid z=1}=0, \quad T_{\mid z=0}=\tilde{R} a, T_{\mid z=1}=0, \quad \mathbf{u}, T \text { are periodic in } \mathbf{x}=(x, y) .
$$

The unitless physical parameters in the problem are the Prandtl number $\operatorname{Pr}$ and Rayleigh numbers $R a$ and $\tilde{R} a$; see Remark 2.3 and [22] for further details concerning this choice of nondimensionalization. The unit vector $\hat{\mathbf{k}}=(0,0,1)$ points in the direction of the gravitational force. The driving noise in (1.2) is given by a collection of independent white noise processes $d W^{k}=d W^{k}(t)$ acting spatially through the functions $\sigma_{k}=\sigma_{k}(x, y, z)$ which form a complete orthogonal basis of eigenfunctions (ordered with respect to eigenvalues) of the Laplace operator on $\mathcal{D}$ supplemented with homogeneous Dirichlet boundary conditions for $z=0,1$ and periodic in $\mathbf{x}=(x, y)$. The stochastic terms in (1.2) have been normalized so that

$$
\sum_{k=1}^{N}\left\|\sigma_{k}\right\|_{L^{2}(\mathcal{D})}^{2}=1,
$$

with the strength of the body forcing expressed in terms of the physical parameters $R a$ and $\tilde{R} a$; see $(2.7)$ below.

Our principal aim here is to establish convergence properties of statistically invariant states of (1.1)-(1.3) to invariant measures of the active scalar equation

$$
\begin{gathered}
-\Delta \mathbf{u}=\nabla p+R a \hat{\mathbf{k}} T, \quad \nabla \cdot \mathbf{u}=0, \\
d T+\mathbf{u} \cdot \nabla T d t=\Delta T d t+\sum_{k=1}^{N} \sigma_{k} d W^{k}
\end{gathered}
$$

in the Large Prandtl number limit that is when $\operatorname{Pr}$ in (1.1) diverges to $\infty$. Here (1.5)-(1.6) is complemented with boundary conditions as in (1.3). Note that $\mathbf{u}$ and $p$ are determined by $T$ according to (1.5). We write this functional dependence as $\mathbf{u}=M(T)$ and denote $L(T)=(M(T), T)$. 
The analysis of convection in the large Prandtl number limit is of basic interest in a variety of physical contexts, most notably in modeling certain portions of the earth's mantle and for convection in gasses under high pressure, where the Prandtl number can reach the order of $10^{24}$, see $[6,12,37]$. It is worth emphasizing that the system (1.5)-(1.6) has very complex dynamics even without stochastic forcing when the Rayleigh number(s) are sufficiently large; see $[1,3,4,6,12,32,37,38,43]$.

\section{Overview of the Main Results}

Let us now present a heuristic version of our main results; for the precise formulation see Theorem 2.2 below. Recall that for any function $F$ and measure $\mu$, the push-forward of $\mu$ under $F$ is given by $F \mu:=\mu \circ F^{-1}$.

Theorem 1.1. Fix any Ra, $\tilde{R} a>0$ and consider (1.1)-(1.3) and (1.5)-(1.6) with $N \geq N(R a, \tilde{R} a)$ independently forced directions in the temperature equation. Then (1.5)-(1.6) possesses a unique ergodic invariant measure $\mu_{\infty}$. Let $\left\{\mu_{P r}\right\}_{P r \geq 1}$ be any sequence of statistically invariant states associated to (1.1)-(1.3) satisfying a uniform exponential moment bound (see (2.13) below noting that $\epsilon:=\frac{1}{\operatorname{Pr}}$ ). ${ }^{1}$ Then $\mu_{P r}$ converges to $L_{\#} \mu_{\infty}$ in a suitable metric. In particular, for any sufficiently regular observable $\phi$ on the $(\mathbf{u}, T)$ phase space,

$$
\left|\int \phi(\mathbf{u}, T) d \mu_{P r}-\int \phi(L(T)) d \mu_{\infty}\right| \leq C(P r)^{-q},
$$

where $C=C(\phi, R a, \tilde{R} a), q=q(R a, \tilde{R} a)>0$ are independent of $\operatorname{Pr}$ and $q$ is independent of $\phi$.

The proof of Theorem 1.1 contains several further results of independent interest. Firstly, we show that the Markovian dynamics of probability laws for the infinite Prandtl system, (1.5)- (1.6) is contractive in a suitable Wasserstein distance; see Theorem 2.2, (2.29) below. Secondly, we demonstrate that the finite time dynamics converge in the limit as $\operatorname{Pr} \rightarrow \infty$.

Note that our results do not rely on the well-posedness of (1.1)-(1.2) or make any assertions concerning the convergence of (1.1)-(1.2) to the formal limit (1.5)-(1.6) for small times. On the other hand, as notable biproduct of our convergence analysis, we derive a well posed approximation of (1.1)-(1.2) up to the initial time $t=0$ which is valid for large values of Pr. See Section 5.3 and Theorem 5.1 below for further details.

It is also worth emphasizing that our proof of Theorem 1.1 applies essentially verbatum to the twodimensional version of (1.1)-(1.3), where the horizontal variable $\mathbf{x}$ is one-dimensional. Here all the statistically invariant states of the full system satisfy the uniform moment bound (2.13). Furthermore, in collaboration with Whitehead [22], the authors have established that with $N=\infty$ and $\operatorname{Pr}=\operatorname{Pr}(\operatorname{Ra}, \tilde{R} a)>0$ sufficiently large, the 2D version of (1.1)-(1.3) possesses a unique ergodic invariant measure $\mu_{P r}$.

An empirical quantity of particular interest in convection is the Nusselt number $N u$, a measurement of the convective heat transfer, which is defined in terms of a statistical average (e.g. a time average) of the observable $\phi_{N u}=\int_{\mathcal{D}} u_{2} T d x .^{2}$ However, in the deterministic case, even in the turbulent regime of Ra $\gg 1$, $N u$ depends on initial condition, both at finite and infinite values of $\mathrm{Pr}$ and it is unclear that $N u$ is continuous at $\operatorname{Pr}=\infty$. We show that the addition of a stochastic perturbation avoids these concerns.

Corollary 1.1. For any $R a, \tilde{R} a>0$ and any sufficiently large $\operatorname{Pr}=\operatorname{Pr}(R a, \tilde{R} a)>0$ the system (1.1)-(1.3) posed in two space dimensions with $N=\infty$ and (1.4) possesses a unique ergodic invariant measure $\mu_{P r}$, and the Nusselt number $N u$ given by (cf. [22, Theorem 1.4])

$$
N u=(N u)_{P r}:=1+\frac{1}{|\mathcal{D}|} \iint_{\mathcal{D}} u_{2} T d x d \mu_{P r}(\mathbf{u}, T)
$$

satisfies

$$
\lim _{P r \rightarrow \infty}(N u)_{P r}=(N u)_{\infty} .
$$

Note that $(\mathrm{Nu})_{\infty}$ is defined by (1.8) relative to the unique ergodic invariant measure $\mu_{\infty}$ of (1.5)-(1.6).

\footnotetext{
${ }^{1}$ Note that usual fundamental difficulties concerning the well-posedness of 3D Navier-Stokes apply to (1.1)-(1.2) and so, following [17], we consider only weak solutions whose laws do not change in time. The uniform exponential moment condition is analogous to a finite energy criterion for weak solutions of the 3D Navier-Stokes equations. In [22] we have established the existence of such states $\mu_{P r}$, see Proposition 2.1 below for a precise restatement. In particular we cannot rule out the existence of a collection $\left\{\mu_{P r}\right\}_{\operatorname{Pr} \in \mathbb{N}}$ which does not satisfy (2.13). Observe that none of these difficulties arise the 2D case.

${ }^{2}$ Here $u_{2}$ represents the vertical velocity component for the $2 \mathrm{D}$ version of $(1.1)-(1.3)$.
} 
Theorem 1.1 and Corollary 1.1 may be seen as complementary to a series of recent works [43-48] which address large Prandtl number asymptotics for the Boussinesq system in a deterministic framework. Here we show that the addition of stochastic terms allows for stronger convergence results, but the proofs require a different framework. In particular, Corollary 1.1 resolves a conjecture of Wang [48] by confirming that stochastic forcing stabilizes the Nusselt number in the infinite Prandtl number limit.

\section{Methods of Analysis}

The starting point of our analysis is to establish a strict contraction property for the Markov semigroup $\left\{P_{t}^{0}\right\}_{t \geq 0}$ associated to the formal limit system (1.5)-(1.6). We show that for, some $t_{*}>0$ sufficiently large and for any probability measures $\mu, \tilde{\mu}$ on the phase space associated with the $T$ component of (1.5)-(1.6), one has

$$
\rho\left(\mu P_{t_{*}}^{0}, \tilde{\mu} P_{t_{*}}^{0}\right) \leq \frac{1}{2} \rho(\mu, \tilde{\mu}),
$$

where $\rho$ is an appropriately chosen Kantorovich-Wasserstein metric. See (2.19) and Theorem 2.2, (i) for a precise formulation.

The bound (1.9) is crucial since it allows us to reduce the proof of the convergence of statistically invariant states in the infinite Prandtl limit to the convergence of solutions on finite time intervals. Indeed, suppose that $\mu_{0}$ is the (unique) invariant measure for (1.5)-(1.6) and for $\varepsilon>0$ let $\mu_{\varepsilon}$ be the $T$ component of any stationary solution of (1.1)-(1.2) with $\varepsilon:=1 /$ Pr. Utilizing the invariance of $\mu_{0}$ and (1.9) we find

$$
\rho\left(\mu_{\varepsilon}, \mu_{0}\right)=\rho\left(\mu_{\varepsilon}, \mu_{0} P_{t_{*}}^{0}\right) \leq \rho\left(\mu_{\varepsilon}, \mu_{\varepsilon} P_{t_{*}}^{0}\right)+\rho\left(\mu_{\varepsilon} P_{t_{*}}^{0}, \mu_{0} P_{t_{*}}^{0}\right) \leq \rho\left(\mu_{\varepsilon}, \mu_{\varepsilon} P_{t_{*}}^{0}\right)+\frac{1}{2} \rho\left(\mu_{\varepsilon}, \mu_{0}\right),
$$

and consequently

$$
\rho\left(\mu_{\varepsilon}, \mu_{0}\right) \leq 2 \rho\left(\mu_{\varepsilon}, \mu_{\varepsilon} P_{t_{*}}^{0}\right) .
$$

By properties of the Wasserstein metric, specifically (2.21), and using the stationarity of the solutions corresponding to $\mu_{\varepsilon}$ we therefore obtain the estimate

$$
\rho\left(\mu_{\varepsilon}, \mu_{0}\right) \leq 2 \mathbb{E} \rho\left(T^{\varepsilon}\left(t_{*}\right), T^{0, \varepsilon}\left(t_{*}\right)\right) .
$$

We have thus bounded the distance between invariant states by the mean distance between solutions at a fixed finite time $t_{*}$. Note that these two solutions satisfy identical initial conditions which are distributed as $\mu_{\varepsilon}$.

Recently the strategy leading to (1.11) has proven effective for establishing the convergence of statistically invariant states for a variety of problems; see [5, 20, 25, 27, 31]. However, in order to implement this approach, one typically faces several major challenges. A first challenge is to prove the contraction estimate (1.9), where the semigroup $\left\{P_{t}^{0}\right\}_{t \geq 0}$ corresponds to (1.5)-(1.6). Moreover, in our setting, it is desirable to lift this contraction property to the extended phase space involving both the velocity $\mathbf{u}$ and temperature components $T$ of our system. This is particularly relevant in view of the physical significance of the Nusselt number, a quantity involving both $\mathbf{u}$ and $T$ as in (1.8). A second challenge to show that $\mathbb{E} \rho\left(T^{\varepsilon}\left(t_{*}\right), T^{0, \varepsilon}\left(t_{*}\right)\right) \rightarrow 0$ as $\varepsilon \rightarrow 0$ in order to take advantage of (1.11). This task requires suitable $\epsilon=\operatorname{Pr}^{-1}$ uniform moment bounds on the stationary statistics $\mu^{\varepsilon}$ and finite time convergence results for solutions in the limit as $\operatorname{Pr} \rightarrow \infty$. As we describe presently the results established here require new ideas in comparison to the aforementioned related works. This is partially due to the presence of non-homogeneous boundary conditions for (1.5)-(1.6) and to the singular nature of the limit from (1.1)-(1.2) to (1.5)-(1.6).

Regarding the first challenge, guided by the classical Doob-Khasminskii Theorem [10, 13, 30] and as encompassed by the more recent developments in $[25,27,29]$, one can establish a contraction of the type (1.9) when the Markov semigroup is smoothing, suitable moment bounds hold, and there is some form of irreducibility in the dynamics. The question of smoothing for the Markov semigroup can be translated to a control problem; see (B.10) below. In our setting, when the number of forced directions $N=N(R a, \tilde{R} a)$ is sufficiently large, an appropriate control can be found through Foias-Prodi type considerations [18]. Since (1.5)-(1.6) may be seen as an advection diffusion system with $\mathbf{u}$ being two derivatives smoother than $T$, 
such a strategy largely repeats the approach used in previous works on the 2D stochastic Navier-Stokes equations $[16,26,27,31]$. On the other hand establishing suitable moment bounds is more delicate due to the non-homogenous boundary conditions imposed in (1.3) and requires a careful use of the maximum principle along with exponential martingale estimates. These bounds have been carried out in our companion work [22]. The main obstacle to demonstrating (1.9) is to establish irreducibility, which does not follow from the approach set out in previous works, e.g. $[8,15,20,21,26]$. This is because the system (1.5)-(1.6) with its stochastic terms removed can have highly non-trivial dynamics, see [6, 12, 37, 38, 43]. We show that, despite this complication, the support of every invariant measure of (1.5)-(1.6) contains the basic conductive state. Indeed we establish with the use of another Foias-Prodi bound that a Girsanov shift of (1.5)-(1.6) converges to the conductive state with positive probability. We then employ moment estimates and stopping time arguments to translate this non-zero probability back to the original system (1.5)-(1.6) yielding the desired irreducibility.

In order to establish convergence of invariant states on the extended phase space, we adapt a methodology from recent joint work of the authors with Friedlander [20] which enhances (1.9) to a "lifted" contraction property with respect to a carefully chosen metric (see Lemma 3.1 below). By invoking this lifted contraction property, and appropriately modifying the argument in (1.10)-(1.11), the convergence of invariant states as $\operatorname{Pr} \rightarrow \infty$ reduces to establishing the convergence of solutions of (1.1)-(1.2) to those of (1.5)-(1.6) at a fixed time $t_{*}>0$, independent of $\varepsilon$, when the initial conditions have the same distribution in temperature only.

The second major challenge regards the convergence of solutions of (1.1)-(1.2) on finite time intervals as $\operatorname{Pr} \rightarrow \infty$ for which we develop a suitable asymptotic analysis. This is a non-trivial task since the small parameter $1 / \operatorname{Pr}$ lies in front of the time derivative terms in (1.1). Moreover the convergence analysis in [43-48] for a deterministic analogue of (1.1)-(1.2) requires significant modification. In particular these references crucially use higher temporal regularity properties which are missing in our stochastic setting. As a substitute we derive a stochastic evolution equation for the velocity component and use martingale properties of associated Itō integrals. Our analysis then takes advantage of uniform moment estimates from [22], some previously unobserved cancellations in certain error terms and delicate stopping time arguments.

Analogous to the results in [43-48] we derive an 'intermediate system', which we refer to as the 'corrector'. We show rigorously that this system approximates the finite Prandtl system in the velocity equation over bounded time intervals up the initial time; cf Theorem 5.1. While this corrector system is of independent interest we also we provide a somewhat simpler and more direct analysis of the convergence of (1.1)-(1.2) to (1.5)-(1.6) which well approximates the infinite Prandtl system after an $O(1 / P r)$ time transient. Indeed, this more direct approach is sufficient for the upper bound in (1.11) since this bound only involves a fixed time $t_{*}>0$.

\section{Manuscript Organization}

The manuscript is organized as follows. In Section 2 we introduce the rigorous mathematical setting of the stochastic Boussinesq equations, (1.1)-(1.3), which serves as a foundation for the rest of the analysis. We also introduce the formalities of the Kantorovich-Wasserstein metric in Section 2.2, and provide a rigorous formulation of our main results in Section 2.3. Section 3 describes core of our strategy that reduces the question of convergence to finite time asymptotics and uniform moment bounds. Section 4 is devoted to establishing the contraction (1.9) for the infinite Prandtl system (1.5)-(1.6). In Section 5 we carry out the finite time convergence analysis. The section concludes with a derivation and analysis of the intermediate corrector system. In Section 6 we establish convergence of the Nusselt number. Finally two Appendices recall various elements essentially contained in previous works that we have used in our analysis. Appendix A is devoted to details for various moment estimates from [22] for a class of drift-diffusion equations which we use to bound (1.5)-(1.6). In Appendix B we outline gradient estimates on the Markov semigroup corresponding to $(1.5)-(1.6)$ which are carried out in a similar fashion to e.g. [26].

\section{Mathematical Preliminaries and Main Results}

We begin our analysis of the stochastic Boussinesq Equations by recalling some details of their mathematical setting. The section concludes with a mathematically precise restatement of Theorem 1.1. Here and below 
we implicitly assume that $C, c, C_{0}$ etc. are constants depending on the domain $\mathcal{D}$ with any other dependency indicated explicitly.

For the forthcoming analysis it is convenient to consider an equivalent homogeneous, form of the stochastic Boussinesq Equations. Introducing the 'small parameter' $\varepsilon=P r^{-1}>0$ and making the change of variable $\theta^{\varepsilon}=T-\tilde{R} a(1-z)$ we rewrite $(1.1)-(1.2) \mathrm{as}^{3}$

$$
\begin{aligned}
& \varepsilon\left(\partial_{t} \mathbf{u}^{\varepsilon}+\mathbf{u}^{\varepsilon} \cdot \nabla \mathbf{u}^{\varepsilon}\right)-\Delta \mathbf{u}^{\varepsilon}=\nabla \tilde{p}^{\varepsilon}+R a \hat{\mathbf{k}} \theta^{\varepsilon}, \quad \nabla \cdot \mathbf{u}^{\varepsilon}=0, \\
& d \theta^{\varepsilon}+\mathbf{u}^{\varepsilon} \cdot \nabla \theta^{\varepsilon} d t=\tilde{R} a \cdot u_{3}^{\varepsilon} d t+\Delta \theta^{\varepsilon} d t+\sum_{k=1}^{N} \sigma_{k} d W^{k},
\end{aligned}
$$

supplemented with the homogenous boundary conditions

$$
\mathbf{u}_{\mid z=0}^{\varepsilon}=\mathbf{u}_{\mid z=1}^{\varepsilon}=0, \quad \theta_{\mid z=0}^{\varepsilon}=\theta_{\mid z=1}^{\varepsilon}=0, \quad \mathbf{u}^{\varepsilon}, \theta^{\varepsilon} \text { are periodic in } \mathbf{x}=(x, y)
$$

Here, in reference to the $\tilde{R} a \cdot u_{3}^{\varepsilon}$ term in (2.2) recall that $\mathbf{u}^{\varepsilon}=\left(u_{1}^{\varepsilon}, u_{2}^{\varepsilon}, u_{3}^{\varepsilon}\right)$. The corresponding infinite Prandtl system $(\varepsilon=0)$ is given by

$$
\begin{aligned}
-\Delta \mathbf{u}^{0} & =\nabla \tilde{p}+R a \hat{\mathbf{k}} \theta^{0}, \quad \nabla \cdot \mathbf{u}^{0}=0 \\
d \theta^{0}+\mathbf{u}^{0} \cdot \nabla \theta^{0} d t & =\tilde{R} a \cdot u_{3}^{0} d t+\Delta \theta^{0} d t+\sum_{k=1}^{N} \sigma_{k} d W^{k}
\end{aligned}
$$

again with initial conditions $\theta^{0}(0)=\theta_{0}^{0}$ and boundary conditions as in (2.3).

Remark 2.1. Notice that we do not prescribe an initial condition for $\mathbf{u}^{0}$ in $(2.4)-(2.5)$ as this component does not satisfy an independent evolution equation. Indeed (2.4)-(2.5) can be rewritten as

$$
d \theta^{0}+\left(M \theta^{0}\right) \cdot \nabla \theta^{0} d t=\tilde{R} a\left(M \theta^{0}\right)_{3} d t+\Delta \theta^{0} d t+\sum_{k=1}^{N} \sigma_{k} d W^{k}
$$

where the constitutive law $M$ recovers $\mathbf{u}$ from $\theta$ according to (2.4) as in (2.9) below.

Remark 2.2. The systems (2.1)-(2.2) and (2.4)-(2.5) can be reformulated in terms of $T=\theta^{\varepsilon}+\tilde{R} a(1-z)$, which satisfies (1.1)-(1.2) or (1.5)-(1.6), respectively, and has boundary conditions (1.3). Our analysis makes use of both of these formulations.

Remark 2.3. As noted above, parameters in the problem are the Prandtl $\left(\operatorname{Pr}=\varepsilon^{-1}\right)$ and Rayleigh numbers $(R a, \tilde{R} a)$, which are unit-less. In terms of basic physical quantities of interest we have that

$$
\varepsilon^{-1}=\operatorname{Pr}=\frac{\nu}{\kappa}, \quad R a=\frac{g \alpha \gamma h^{5 / 2}}{\nu \kappa^{3 / 2}}, \quad \tilde{R} a=\frac{\sqrt{\kappa h}\left(T_{b}-T_{t}\right)}{\gamma},
$$

where $\nu$ is the kinematic viscosity, $\kappa$ the thermal diffusivity, $g$ the gravitational constant, $\alpha$ the coefficient of thermal expansion, $h$ the distance between the confining plates, $T_{b}-T_{t}$ the temperature differential, and $\gamma=\mathcal{H} / \rho c$ the intensity $\mathcal{H}$ of the volumetric heat flux normalized by the density $\rho$ and specific heat $c$ of the fluid. We refer the interested reader to [22], where the dimensionless form of the stochastically driven Boussinesq equations is derived.

\subsection{Functional Setting of the Boussinesq Equations}

We next define the phase space for the Boussinesq equations, which is very close to the classical framework for the Navier-Stokes equations; see e.g. [7, 40] for further details.

\footnotetext{
${ }^{3}$ Note that we have implicitly modified the pressure in $(2.1)$ by $\operatorname{Ra} \tilde{R} a\left(z-\frac{1}{2} z^{2}\right)$ since $(1-z) \hat{\mathbf{k}}=\nabla\left(z-\frac{1}{2} z^{2}\right)$.
} 
We define $\mathbf{H}:=H_{1} \times H_{2}$ as the phase space for (2.1)-(2.3), where

$$
\begin{aligned}
& H_{1}:=\left\{\mathbf{u} \in\left(L^{2}(\mathcal{D})\right)^{3}: \nabla \cdot \mathbf{u}=0, \mathbf{u} \cdot \mathbf{n}_{\mid z=0,1}=0, \mathbf{u} \text { is periodic in } \mathbf{x}\right\}, \\
& H_{2}:=\left\{\theta \in L^{2}(\mathcal{D}): \theta \text { is periodic in } \mathbf{x}\right\}
\end{aligned}
$$

and we denote by $H=H_{2}$ the phase space for (2.4)-(2.5). The spaces $\mathbf{H}$ and $H$ are endowed with the standard $L^{2}$-norm and we denote each of them by $\|\cdot\|$ as the appropriate meaning will be clear from the context. ${ }^{4}$ All other norms are written as $\|\cdot\|_{\mathbb{X}}$ below for a given space $\mathbb{X}$. We define $H^{1}$ type spaces as

$$
\begin{aligned}
& V_{1}:=\left\{\mathbf{u} \in\left(H^{1}(\mathcal{D})\right)^{3}: \nabla \cdot \mathbf{u}=0, \mathbf{u}_{\mid z=0,1}=0, \mathbf{u} \text { is periodic in } \mathbf{x}\right\}, \\
& V_{2}:=\left\{\theta \in H^{1}(\mathcal{D}): \theta_{\mid z=0,1}=0, \theta \text { is periodic in } \mathbf{x}\right\} .
\end{aligned}
$$

Let $\mathbf{V}=V_{1} \times V_{2}$ and $V=V_{2}$. We will sometimes consider the $L^{p}(\mathcal{D})$ spaces of $p$-integrable functions for $p \in[1, \infty]$ and endow these spaces with their standard norms.

In what follows we frequently project or lift the dynamics to account for the phase space mismatch between (2.1)-(2.2) and (2.4)-(2.5). We define

$$
\Pi: \mathbf{H} \rightarrow H_{2} \text { to be the projection onto the } \theta \text { component of } \mathbf{H} \text {. }
$$

Associated with the limit system (2.4)-(2.5) we have the constitutive law

$$
M(\theta)=R a A^{-1} P \theta \hat{k}
$$

where $A$ is the Stokes operator and $P$ the Leray projector. In other words $\mathbf{u}=M(\theta)$ is the solution of

$$
-\Delta \mathbf{u}=\nabla \tilde{p}+R a \hat{\mathbf{k}} \theta, \quad \nabla \cdot \mathbf{u}=0
$$

see Section 5.1 and in particular (5.1) below. We define the 'lifting map' $L: H \rightarrow \mathbf{H}$ from the temperature component to the extended phase space

$$
L(\theta)=(M(\theta), \theta) .
$$

Finally we denote $\operatorname{Pr}(\mathbb{X})$ as the space of Borel probability measures on a given complete metrizable space $\mathbb{X}$, typically $H, \mathbf{H}$ etc. For $\mu \in \operatorname{Pr}(\mathbf{H})$, we take $\Pi \mu(\cdot)=\mu\left(\Pi^{-1}(\cdot)\right)$ to be the push-forward of $\mu$ by $\Pi$. Similarly $L \mu$ is the push-forward of $\mu$ by $L$ when $\mu \in \operatorname{Pr}(H)$.

We have the following general results concerning the existence and uniqueness of solutions of (2.1)-(2.3) and (2.4)-(2.5):

Proposition 2.1 (Existence, Uniqueness and Continuous Dependence of Solutions on Data). Fix any values $R a, \tilde{R} a>0$.

(i) For every $\epsilon>0$ and any given $\mu^{0} \in \operatorname{Pr}(\mathbf{H})$ with $\int\left(\|\mathbf{u}\|^{2}+\|\theta\|^{2}\right) d \mu^{0}(\mathbf{u}, \theta)<\infty$ there exists a stochastic basis $\mathcal{S}=\left(\Omega, \mathcal{F},\left\{\mathcal{F}_{t}\right\}_{t \geq 0}, \mathbb{P}, W\right)$ upon which is defined an $\mathbf{H}$-valued stochastic process $\left(\mathbf{u}^{\varepsilon}, \theta^{\varepsilon}\right)$ with the regularity

$$
\left(\mathbf{u}^{\varepsilon}, \theta^{\varepsilon}\right) \in L^{2}\left(\Omega ; L_{l o c}^{2}([0, \infty) ; \mathbf{V}) \cap L_{l o c}^{\infty}([0, \infty) ; \mathbf{H})\right),
$$

which is weakly continuous in $\mathbf{H}$, adapted to $\left\{\mathcal{F}_{t}\right\}_{t \geq 0}$, satisfies $(2.1)-(2.2)$ weakly and such that $\left(\mathbf{u}^{\varepsilon}(0), \theta^{\varepsilon}(0)\right)$ is distributed as $\mu^{0}$. We say that such a pair $\left(\mathcal{S},\left(\mathbf{u}^{\varepsilon}, \theta^{\varepsilon}\right)\right)$ is a weak-martingale solution of (2.1)-(2.3). If, for some $p \geq 2$, and $\eta>0$,

$$
\int_{\mathbf{H}} \exp \left(\eta\left(\|\mathbf{u}\|^{2}+\|\theta\|_{L^{p}}^{2}\right)\right) d \mu^{0}(\mathbf{u}, \theta)<\infty,
$$

there exists $\eta_{0}>0$ and a weak martingale solution $\left(\mathcal{S},\left(\mathbf{u}^{\varepsilon}, \theta^{\varepsilon}\right)\right)$ such that

$$
\mathbb{E} \exp \left(\eta_{0}\left(\sup _{s \in[0, t]}\left(\left\|\mathbf{u}^{\varepsilon}\right\|^{2}+\left\|\theta^{\varepsilon}\right\|_{L^{p}}^{2}\right)+\int_{0}^{t}\left(\left\|\nabla \mathbf{u}^{\varepsilon}\right\|^{2}+\left\|\nabla \theta^{\varepsilon}\right\|^{2}\right) d s\right)\right) \leq C<\infty
$$

for each $t>0$, where $C>0$ is a constant independent of $\varepsilon \in(0,1]$.

\footnotetext{
${ }^{4}$ Below will also consider the weighted metrics $(2.22)$, (2.24) which generate an equivalent topology on $\mathbf{H}$ and $H$ but are more suitable for the convergence of measures in the associated Wasserstein metric.
} 
(ii) Additionally, for any $\varepsilon>0$, there exists a martingale solution $\left(\mathcal{S},\left(\mathbf{u}_{S}^{\varepsilon}, \theta_{S}^{\varepsilon}\right)\right)$ of $(2.1)-(2.2)$ which is stationary in time, meaning that the law of the solution is independent of time. These stationary solutions $\left(\mathcal{S},\left(\mathbf{u}_{S}^{\varepsilon}, \theta_{S}^{\varepsilon}\right)\right)$ may be chosen in such a way that, for any $p \geq 2$ there is an $\eta=\eta(p, R a, \tilde{R} a)>0$, for which

$$
\sup _{1 \geq \varepsilon>0} \int_{\mathbf{H}} \exp \left(\eta\left(\|\mathbf{u}\|^{2}+\|\theta\|_{L^{p}}^{2}\right)\right) d \mu_{\varepsilon}(\mathbf{u}, \theta)=C_{0}<\infty
$$

where $\mu_{\varepsilon}(\cdot)=\mathbb{P}\left(\left(\mathbf{u}_{S}^{\varepsilon}(t), \theta_{S}^{\varepsilon}(t)\right) \in \cdot\right)$ for any fixed $t \geq 0$.

(iii) Now consider the case when $\varepsilon=0$. Fix a stochastic basis $\mathcal{S}$ and any $\mathcal{F}_{0}$-measurable random variable $\theta_{0} \in L^{2}(\Omega, H)$. Then there exists a unique process $\theta^{0}$ with

$$
\theta^{0} \in L^{2}\left(\Omega ; L_{l o c}^{2}([0, \infty) ; V) \cap C([0, \infty) ; H)\right),
$$

which is $\mathcal{F}_{t}$-adapted, weakly solves $(2.4)-(2.5)$, and satisfies the initial condition $\theta^{0}(0)=\theta_{0}$.

(iv) For a given stochastic basis $\mathcal{S}$ and each $\theta_{0} \in H$ denote $\theta^{0}\left(\cdot, \theta_{0}, W\right)$ as the unique corresponding stochastic process satisfying (2.4)-(2.5) with (2.14). We have that $\theta_{0} \mapsto \theta^{0}\left(t, \theta_{0}, W\right)$ is Fréchet differentiable in $\theta_{0} \in H$ for any $t \geq 0$ and any fixed realization $W(\cdot)=W(\cdot, \omega)$. On the other hand $W \mapsto \theta^{0}\left(t, \theta_{0}, W\right)$ is Fréchet differentiable in $W$ from $C_{0}\left([0, t], \mathbb{R}^{N}\right)$ to $H$ for each fixed $\theta_{0} \in H$ and $t>0$.

These results are standard for a systems like (2.1)-(2.3) and (2.4)-(2.5); see e.g. [9, 11, 17, 23]. The only novelty in view of existing methods is the uniform moment bound (2.13). The existence of such a collection of solutions is established using the maximum principle and exponential moment bounds in the companion work [22]; cf. Appendix A below.

The Markovian framework for (2.4)-(2.5) is defined as follows. The transition functions are given by

$$
P_{t}^{0}\left(\theta_{0}, A\right):=\mathbb{P}\left(\theta^{0}\left(t, \theta_{0}\right) \in A\right), \quad t \geq 0, \theta_{0} \in H, A \in \mathcal{B}(H),
$$

where $\mathcal{B}(H)$ denotes the Borel sets of $H$, and the associated semigroup is given by

$$
P_{t}^{0} \phi\left(\theta_{0}\right):=\mathbb{E} \phi\left(\theta^{0}\left(t, \theta_{0}\right)\right), \quad t \geq 0, \phi \in M_{b}(H),
$$

where $M_{b}(H)$ is the set of bounded measurable functions on $H$. In view of the continuous dependence on initial conditions the semigroup $\left\{P_{t}^{0}\right\}_{t>0}$ is Feller, that is, it maps the set of continuous bounded functions on $H, C_{b}(H)$, to itself. This semigroup acts on Borelian probability measures $\mu$ according to

$$
\mu P_{t}^{0}(A)=\int_{H} P_{t}^{0}(\theta, A) d \mu(\theta), \quad A \in \mathcal{B}(H)
$$

A measure $\mu \in \operatorname{Pr}(H)$ is said to be invariant with respect to $\left\{P_{t}^{0}\right\}_{t \geq 0}$ if $\mu P_{t}^{0}=\mu$ for all $t \geq 0$. Recall that in three space dimensions the Markovian framework for the full system with $\varepsilon>0$ cannot be implemented due to a lack of global well-posedness.

As an immediate consequence of bounds in Appendix A and the Krylov-Bogolyubov averaging technique we have

Lemma 2.1. Under the assumptions of Proposition 2.1 and for any Ra, Ra there exists an invariant measure $\mu_{0}$ of the Markov semigroup $P_{t}^{0}$. Moreover for any such measure

$$
\int_{H} \exp \left(\eta\|\theta\|_{L^{p}}^{2}\right) d \mu_{0}(\theta) \leq C_{0}<\infty
$$

for any $p \geq 2$ and any suitably small $\eta=\eta(p, R a, \tilde{R} a)$. 


\subsection{Wasserstein Distance, Weighted Metrics and Associated Observables}

We next recall the general setting of the Kantorovich-Wasserstein distance in which we establish our convergence results. We then introduce weighted metrics on $H$ and $\mathbf{H}$ along with some associated classes of observable which are used to measure distances between measures in the analysis below.

Let $(\mathbb{X}, \rho)$ be a complete metric space and take $\operatorname{Pr}_{1}(\mathbb{X}, \rho)$ to be the set of Borel probability measures $\mu$ on $\mathbb{X}$ with $\int \rho_{\eta}(0, \theta) d \mu(\theta)<\infty$. On $\operatorname{Pr}_{1}(\mathbb{X}, \rho)$ we define the Kantorovich-Wasserstein metric, relative to $\rho$, equivalently as ${ }^{5}$

$$
\rho(\mu, \tilde{\mu}):=\sup _{\|\phi\|_{L i p, \rho} \leq 1}\left|\int_{\mathbb{X}} \phi(\theta) d \mu(\theta)-\int_{\mathbb{X}} \phi(\theta) d \tilde{\mu}(\theta)\right|=\inf _{\Gamma \in \mathcal{C}(\mu, \tilde{\mu})} \int_{\mathbb{X} \times \mathbb{X}} \rho(\theta, \tilde{\theta}) d \Gamma(\theta, \tilde{\theta}),
$$

where

$$
\|\phi\|_{L i p, \rho}:=\sup _{\theta \neq \tilde{\theta}} \frac{|\phi(\theta)-\phi(\tilde{\theta})|}{\rho(\theta, \tilde{\theta})}
$$

for $\phi: \mathbb{X} \rightarrow \mathbb{R}$, and $\mathcal{C}(\mu, \tilde{\mu})$ is the collection of Borel probability measures $\Gamma$ in $\operatorname{Pr}(\mathbb{X} \times \mathbb{X})$ with $\mu, \tilde{\mu}$ as its marginals. Hence, the last term in (2.19) is equivalent to

$$
\rho(\mu, \tilde{\mu})=\inf \mathbb{E} \rho(X, Y),
$$

where the infimum is taken over all $\mathbb{X}$-valued random variables $X, Y$ distributed as $\mu, \tilde{\mu}$ respectively. See e.g. $[14,42]$ for further background on these metrics.

Specializing to our current setting, the following metrics on $H$ and $\mathbf{H}$ prove useful for measuring the distance between the laws of solutions of (2.1)-(2.2) and (2.4)-(2.5). Following e.g. [27] we introduce, for $\eta>0$, the weighted metric on $H$ as

$$
\rho_{\eta}(\theta, \tilde{\theta})=\inf _{\substack{\gamma \in C^{1}([0,1] ; H) \\ \gamma(0)=\theta, \gamma(1)=\tilde{\theta}}} \int_{0}^{1} \exp \left(\eta\|\gamma\|^{2}\right)\left\|\gamma^{\prime}(s)\right\| d s,
$$

for any $\theta, \tilde{\theta} \in H$. Notice that

$$
\|\theta-\tilde{\theta}\| \leq \rho_{\eta}(\theta, \tilde{\theta}) \leq \exp \left(2 \eta\left(\|\theta\|^{2}+\|\tilde{\theta}\|^{2}\right)\right)\|\theta-\tilde{\theta}\|,
$$

for $\theta, \tilde{\theta} \in H$. For the extended phase space $\mathbf{H}$, similarly to our recent work [20], we take

$$
\tilde{\rho}_{\eta}((\mathbf{u}, \theta),(\tilde{\mathbf{u}}, \tilde{\theta}))=\|\mathbf{u}-\tilde{\mathbf{u}}\|_{H^{1}}+\rho_{\eta}(\theta, \tilde{\theta}),
$$

again defined for any $\eta>0$.

For the statement of the main results we consider the following class of 'observables'

$$
\mathcal{V}(\mathbf{H})=\mathcal{V}_{\eta}(\mathbf{H}):=\left\{\phi \in C^{1}(\mathbf{H}):[\phi]_{\eta}<\infty\right\}
$$

where the semi-norm $[\cdot]_{\eta}$ is given by

$$
[\phi]_{\eta}:=\sup _{(u, \theta) \in \mathbf{H}}\left[\sup _{\zeta \in \mathbf{H},\|\zeta\|=1}\left|\nabla_{u} \phi(u, \theta) \cdot \zeta\right|+\exp (-\eta\|\theta\|) \sup _{\xi \in H,\|\xi\|=1}\left|\nabla_{\theta} \phi(u, \theta) \cdot \xi\right|\right] .
$$

Note that, as in [27, Proposition 4.1],

$$
\|\phi\|_{L i p, \tilde{\rho}_{\eta}} \leq C[\phi]_{\eta},
$$

for any $\phi \in C^{1}(\mathbf{H})$ with the constant $C$ independent of $\phi$.

\footnotetext{
${ }^{5}$ Here note slight abuse of notation wherein we denote both the underlying metric and its Wasserstein by $\rho$; the meaning of $\rho$ will be clear from context in what follows.
} 


\subsection{Statement of the Main Results}

We now precisely formulate the main results of this work on the convergence of solutions when $\operatorname{Pr} \rightarrow \infty$. We begin with the following finite time convergence result:

Theorem 2.1. For each $\varepsilon \in(0,1)$, let $\left(\mathbf{u}^{\varepsilon}, \theta^{\varepsilon}\right)$ with its associated stochastic basis $\mathcal{S}$ be a Martingale solution of (2.1)-(2.2) in the sense of Proposition 2.1. Relative to this $\mathcal{S}$, let $\theta^{0}$ be a solution of (2.4)-(2.5). Suppose there exists $C_{0}, \eta>0$ such that ${ }^{6}$

$$
\sup _{\varepsilon>0} \mathbb{E}\left[\exp \left(\eta\left(\left\|\mathbf{u}^{\varepsilon}(0)\right\|^{2}+\left\|\theta^{\varepsilon}(0)\right\|_{L^{3}}^{2}+\left\|\theta^{0}(0)\right\|_{L^{3}}^{2}\right)\right)\right] \leq C_{0}<\infty
$$

and suppose that $\left(\mathbf{u}^{\varepsilon}, \theta^{\varepsilon}\right)$ maintains (2.13). Then, for each $t>0$, there exists $\gamma_{0}>0, C>0$ such that

$$
\begin{aligned}
& \mathbb{E}\left(\sup _{s \in[0, t]}\left\|\theta^{\varepsilon}(s)-\theta^{0}(s)\right\|^{p}\right.\left.+\int_{0}^{t}\left\|\mathbf{u}^{\varepsilon}(s)-M\left(\theta^{0}\right)(s)\right\|_{H^{1}}^{2} d s\right) \\
& \leq C\left(\varepsilon^{\gamma}+\left(\mathbb{E}\left\|\theta^{\varepsilon}(0)-\theta^{0}(0)\right\|^{2}+\varepsilon \mathbb{E}\left\|\mathbf{u}^{\varepsilon}(0)-M\left(\theta^{0}\right)(0)\right\|^{2}\right)^{\gamma}\right),
\end{aligned}
$$

for each $0<\gamma \leq \gamma_{0}$ and any $p>\gamma$. Here the constants $C=C\left(p, \eta, R a, \tilde{R} a, C_{0},\|\sigma\|_{L^{3}}, t\right)$ and $\gamma_{0}=$ $\gamma_{0}\left(\eta, R a, \tilde{R} a, C_{0},\|\sigma\|_{L^{3}}, t\right)$ are independent of $\varepsilon>0$ and depends on the initial conditions only through $C_{0}$.

The proof of Theorem 2.1 is established in Section 5.2.

Remark 2.4. It is worth noting that since $\left(\mathbf{u}^{\varepsilon}, \theta^{\varepsilon}\right)$ are only Martingale solutions the associated stochastic bases $\mathcal{S}$ are not unique and could in fact vary as a function of $\varepsilon$; that is, we cannot assume that these solutions are all defined relative to the same stochastic basis. Similar remarks apply to the bound (2.30) below. However, crucially, in both (2.27) (2.30) the constants do not depend on the choice of bases. Thus, since this subtlety does not cause any trouble in what follows, we shall henceforth suppress this technical point in order to avoid notational confusion.

We next state our results regarding the convergence of statistically invariant states to the unique invariant measure of the formal limit system (cf. Theorem 1.1).

Theorem 2.2. Let $\left\{P_{t}^{0}\right\}_{t \geq 0}$ be the Markov semigroup associated to (2.4)-(2.5) defined in (2.16). There exists $N_{0}>0$ and $\eta_{0}>0$ depending only on $R a$ and $\tilde{R} a$ such that if $N \geq N_{0}$, where $N_{0}$ is the number of stochastically forced modes in (2.4)-(2.5), then the following bounds hold:

(i) For some $\gamma, C>0$ depending only on $R a$ and $\tilde{R} a$

$$
\rho_{\eta}\left(\mu P_{t}^{0}, \tilde{\mu} P_{t}^{0}\right) \leq C \exp (-\gamma t) \rho_{\eta}(\mu, \tilde{\mu})
$$

for any $\mu, \tilde{\mu} \in \operatorname{Pr}_{1}\left(H, \rho_{\eta}\right), \eta \in\left(0, \eta_{0}\right)$ and every $t \geq 0$, where $\rho_{\eta}$ is defined in (2.19). In particular, there exists a unique ergodic invariant measure $\mu_{0} \in \operatorname{Pr}_{1}\left(H, \rho_{\eta}\right)$ of $(2.4)-(2.5)$.

(ii) Suppose that $\left\{\mu_{\varepsilon}\right\}_{\varepsilon>0}$ is any collection of measures corresponding to stationary martingale solutions of (2.1)-(2.3) and satisfying the uniform bound (2.13) for any $\eta \in\left(0, \eta_{0}\right)$ and some $p \geq 3$. Let $\mu_{0}$ be the unique invariant measure of (2.4)-(2.5). Then, there exists $\tilde{q}=\tilde{q}(R a, \tilde{R} a), \tilde{C}=\tilde{C}(R a, \tilde{R} a)$, independent of $\varepsilon>0$, such that

$$
\tilde{\rho}_{\eta}\left(\mu_{\varepsilon}, L \mu_{0}\right) \leq \tilde{C} \varepsilon^{\tilde{q}}
$$

for every $\varepsilon>0$. Consequently for the stationary processes $\left(\mathbf{u}_{S}^{\varepsilon}, \theta_{S}^{\varepsilon}\right)$ and $\theta_{S}^{0}$, distributed as $\mu_{\varepsilon}$ and $\mu_{0}$, respectively,

$$
\left|\mathbb{E}\left(\phi\left(\mathbf{u}_{S}^{\varepsilon}, \theta_{S}^{\varepsilon}\right)-\phi\left(L \theta_{S}^{0}\right)\right)\right| \leq \tilde{C}[\phi]_{\eta} \varepsilon^{\tilde{q}}
$$

for any $\phi \in \mathcal{V}(\mathbf{H})$.

\footnotetext{
${ }^{6}$ Although we can relax the assumption on the initial velocity field to $q$ th moment bounds for some $q \geq 4$, we have opted to impose an exponential moment condition for simplicity of presentation.
} 
The proof of (i) is carried out in Section 4 with some technical details relegated to Appendices A and B. In Section 3 we describe a general strategy which shows that, under the conditions of Theorem 2.2, (2.29) follows from (2.28) and (2.27).

We conclude this section by making several important remarks.

\section{Remark 2.5.}

(i) Assertions of Theorem 2.2 also hold in two space dimensions and in addition one can show that (2.1)(2.3) has a well defined Markov semigroup. Thus, any statistically invariant state corresponds to an invariant measure of the associated semigroup. This allows us to show in [22] that the $\varepsilon$-independent exponential moment bounds in (2.13) hold for all invariant measures.

(ii) In 3D, the existence of a sequence of statistically invariant states of (2.1)-(2.3) satisfying the uniform moment bound (2.13) is established in the companion work [22]. However we have not been able to show that every sequence of invariant states have such (uniform) exponential moments.

(iii) In Section 5 we also derive a 'corrector' system which well approximates the dynamics of the velocity field of the full system (2.1)-(2.2) up to the initial time for large values of $\operatorname{Pr}$ or equivalently small $\varepsilon>0$. See Theorem 5.1 below for further details. Note however that this more refined version of (2.27) is not needed in order to achieve (2.29), (2.30).

\section{Reduction to Finite Time Dynamics}

In this section we describe a general strategy for reducing the convergence of measures to finite time asymptotics and uniform moment bounds when the formal limit system satisfies a suitable mixing condition as in (2.28). To fix ideas we assume the conditions of Theorem 2.2 throughout this section. Also, we assume that both (2.28), (2.27) hold; we establish these bounds rigorously below in Sections 4, 5 respectively. The reader should note that the presented method is flexible and can be applied in a variety of settings. See, for example, [20, 25, 27, 31].

We adapt some ideas from our recent work [20, Section 5] to the present setting. For $\eta>0$ take

$$
\rho_{\eta}^{*}(\theta, \tilde{\theta})=\tilde{\rho}_{\eta}(L(\theta), L(\tilde{\theta})),
$$

where $\tilde{\rho}_{\eta}$ is defined in (2.24) and recall that $L$ is the lifting operator given in (2.10). It is not hard to show that the metrics $\rho_{\eta}$ and $\rho_{\eta}^{*}$ are equivalent (see [20] for details), and consequently the associated Wasserstein metrics on $H$ are also equivalent. Then from (2.28) we obtain the following result, see [20, Corollary 5.4] and surrounding commentary for further details.

Lemma 3.1. Under the same conditions as Theorem 2.2 (i), we have

$$
\tilde{\rho}_{\eta}\left(L\left(\mu P_{t}^{0}\right), L\left(\tilde{\mu} P_{t}^{0}\right)\right) \leq C e^{-\gamma t} \tilde{\rho}_{\eta}(L(\mu), L(\tilde{\mu}))
$$

for any $\mu, \tilde{\mu} \in \operatorname{Pr}_{1}\left(H, \rho_{\eta}\right)$, and every $t \geq 0$.

Using (3.1) choose $t^{*}>0$ to guarantee that

$$
\tilde{\rho}_{\eta}\left(L\left(\mu P_{t^{*}}^{0}\right), L\left(\tilde{\mu} P_{t^{*}}^{0}\right)\right) \leq \frac{1}{2} \tilde{\rho}_{\eta}(L(\mu), L(\tilde{\mu})) .
$$

By the invariance of $\mu_{0}$

$$
\begin{aligned}
\tilde{\rho}_{\eta}\left(\tilde{\mu}, L \mu_{0}\right) & \leq \tilde{\rho}_{\eta}\left(\tilde{\mu}, L\left((\Pi \tilde{\mu}) P_{t+t^{*}}^{0}\right)\right)+\tilde{\rho}_{\eta}\left(L\left((\Pi \tilde{\mu}) P_{t+t^{*}}^{0}\right), L\left(\mu_{0} P_{t+t^{*}}^{0}\right)\right) \\
& \leq \tilde{\rho}_{\eta}\left(\tilde{\mu}, L\left((\Pi \tilde{\mu}) P_{t+t^{*}}^{0}\right)\right)+\frac{1}{2}\left[\tilde{\rho}_{\eta}\left(L\left((\Pi \tilde{\mu}) P_{t}^{0}\right), \tilde{\mu}\right)+\tilde{\rho}_{\eta}\left(\tilde{\mu}, L \mu_{0}\right)\right]
\end{aligned}
$$


for any $t \geq 0$ and any other measure $\tilde{\mu} \in \operatorname{Pr}(\mathbf{H})$. Here recall that $\Pi$ is the projection operator defined in (2.8). Rearranging, taking a time average we obtain

$$
\begin{aligned}
\tilde{\rho}_{\eta}\left(\tilde{\mu}, L \mu_{0}\right) & \leq \frac{2}{t^{*}} \int_{0}^{t^{*}}\left[\tilde{\rho}_{\eta}\left(\tilde{\mu}, L\left((\Pi \tilde{\mu}) P_{t+t^{*}}^{0}\right)\right)+\tilde{\rho}_{\eta}\left(\tilde{\mu}, L\left((\Pi \tilde{\mu}) P_{t}^{0}\right)\right)\right] d t \\
& =\frac{2}{t^{*}} \int_{0}^{2 t^{*}} \tilde{\rho}_{\eta}\left(\tilde{\mu}, L\left((\Pi \tilde{\mu}) P_{t}^{0}\right)\right) d t .
\end{aligned}
$$

With (3.3) now in hand, we consider an sequence of stationary Martingale solutions $\left\{\left(\mathbf{u}_{S}^{\varepsilon}, \theta_{S}^{\varepsilon}\right)\right\}_{\varepsilon}>0$ and take $\left\{\mu_{\varepsilon}\right\}_{\varepsilon>0} \subset \operatorname{Pr}(\mathbf{H})$ to be the corresponding collection of stationary measures. We suppose that $\left\{\mu_{\varepsilon}\right\}_{\varepsilon>0}$ satisfies the uniform moment condition (2.13) as in Proposition 2.1, (ii). We also denote $\theta_{S}^{0, \varepsilon}$ (and $M\left(\theta_{S}^{0, \varepsilon}\right)$ ) the solution of $(2.4),(2.5)$ with the initial condition $\theta^{\varepsilon}(0)$ so that, for every $t>0$, the law of $\theta_{S}^{0, \varepsilon}(t)$ is $\left(\Pi \mu_{\varepsilon}\right) P_{t}^{0}$. Consequently, (2.21), (2.24) yield

$$
\tilde{\rho}_{\eta}\left(\mu_{\varepsilon}, L\left(\left(\Pi \mu_{\varepsilon}\right) P_{t}^{0}\right)\right) \leq \mathbb{E}\left\|\mathbf{u}_{S}^{\varepsilon}(t)-M\left(\theta_{S}^{0, \varepsilon}(t)\right)\right\|_{H^{1}}+\mathbb{E} \rho_{\eta}\left(\theta_{S}^{\varepsilon}(t), \theta_{S}^{0, \varepsilon}(t)\right),
$$

where recall $M$ is defined as in (2.9). By (2.23) one has, for any $q>0$,

$$
\begin{aligned}
\mathbb{E} \rho_{\eta}\left(\theta_{S}^{\varepsilon}(t), \theta_{S}^{0, \varepsilon}(t)\right) & \left.\leq \mathbb{E}\left(\exp \left(2 \eta\left(\left\|\theta_{S}^{\varepsilon}(t)\right\|^{2}+\left\|\theta_{S}^{0, \varepsilon}(t)\right\|^{2}\right)\right) \| \theta_{S}^{\varepsilon}(t)-\theta_{S}^{0, \varepsilon}(t)\right) \|\right) \\
& \leq C \mathbb{E}\left(\exp \left(3 \eta\left(\left\|\theta_{S}^{\varepsilon}(t)\right\|^{2}+\left\|\theta_{S}^{0, \varepsilon}(t)\right\|^{2}\right)\right)\left\|\theta_{S}^{\varepsilon}(t)-\theta_{S}^{0, \varepsilon}(t)\right\|^{q / 2}\right) \\
& \left.\leq C\left(\mathbb{E} \exp \left(12 \eta\left\|\theta_{S}^{\varepsilon}(t)\right\|^{2}\right) \cdot \mathbb{E} \exp \left(12 \eta\left\|\theta_{S}^{0, \varepsilon}(t)\right\|^{2}\right)\right)\right)^{1 / 4}\left(\mathbb{E}\left\|\theta_{S}^{\varepsilon}(t)-\theta_{S}^{0, \varepsilon}(t)\right\|^{q}\right)^{1 / 2} .
\end{aligned}
$$

Using (A.4) with $p=2$ we obtain

$$
\mathbb{E} \rho_{\eta}\left(\theta_{S}^{\varepsilon}(t), \theta_{S}^{0, \varepsilon}(t)\right) \leq C\left(\mathbb{E} \exp \left(96 \eta\left\|\theta_{S}^{\varepsilon}(0)\right\|^{2}\right)\right)^{1 / 2}\left(\mathbb{E}\left\|\theta_{S}^{\varepsilon}(t)-\theta_{S}^{0, \varepsilon}(t)\right\|^{q}\right)^{1 / 2} .
$$

Finally combining (3.3) with (3.4), (3.5) we obtain

$$
\begin{aligned}
\tilde{\rho}_{\eta}\left(\mu_{\varepsilon}, L \mu_{0}\right) \leq & C \mathbb{E} \int_{0}^{2 t *}\left\|\mathbf{u}_{S}^{\varepsilon}(t)-M\left(\theta_{S}^{0, \varepsilon}(t)\right)\right\|_{H^{1}} d t \\
& +C\left(\mathbb{E} \exp \left(96 \eta\left\|\theta_{S}^{\varepsilon}(0)\right\|^{2}\right)\right)^{1 / 2} \sup _{t \in\left[0,2 t^{*}\right]}\left(\mathbb{E}\left\|\theta_{S}^{\varepsilon}(t)-\theta_{S}^{0, \varepsilon}(t)\right\|^{q}\right)^{1 / 2},
\end{aligned}
$$

which holds for any $t^{*}>0$ such that (3.2) holds.

With (3.6) established we conclude this section by detailing the proof of Theorem 2.2, (ii) up to the supporting results proven in Sections 4 and 5.

Proof of Theorem 2.2, (ii). The inequality (2.28) implies (3.1) which in turn implies the bound (3.6). Applying (2.27) with $\left(\mathbf{u}^{\varepsilon}, \theta^{\varepsilon}\right)=\left(\mathbf{u}_{S}^{\varepsilon}, \theta_{S}^{\varepsilon}\right)$ and $\theta^{0}=\theta_{S}^{0, \varepsilon}$, noting $\theta_{S}^{0, \varepsilon}(0)=\theta^{\varepsilon}(0)$, and recalling the assumed bound (2.13) we infer (2.29). To prove (2.30), let $C$ be as in (2.25). Since the Lipschitz norm, with metric $\tilde{\rho}_{\eta}$ of $\psi:=\phi / C[\phi]_{\eta}$ is at most one, then, by $(2.19)$

$$
\left|\mathbb{E}\left(\psi\left(\mathbf{u}_{S}^{\varepsilon}, \theta_{S}^{\varepsilon}\right)-\psi\left(L \theta_{S}^{0}\right)\right)\right|=\left|\int_{\mathbf{H}} \psi(\mathbf{u}, \theta) \mu_{\varepsilon}(\mathbf{u}, \theta)-\int_{H} \psi(\mathbf{u}, \theta) L\left(\mu_{0}\right)(\theta, \mathbf{u})\right| \leq \tilde{\rho}_{\eta}\left(\mu_{\varepsilon}, L \mu_{0}\right),
$$

and the result follows from (2.29). The proof is complete.

\section{Contraction in the Wasserstein Distance for the Infinite Prandtl System}

In this section we establish some properties of the infinite Prandtl system (2.4)-(2.5), which provide a sufficient condition for proving Theorem 2.2 (i) as a consequence of a general result in [27, Theorem 3.4]. These properties are summarized as follows: 
Proposition 4.1. There exist $\eta_{0}>0$ and $N_{0}$, depending only on $R a, \tilde{R} a$, such that for any $0<\eta<\eta_{0}$, whenever the number of forced modes $N$ exceeds $N_{0}$, we have

(a) Lyapunov structure: For all $t^{*}>0$, there exists $C_{1}=C_{1}\left(t^{*}, \eta\right)$ such that for each $\theta_{0}^{0} \in H$ and every $t \in\left[0, t^{*}\right]$

$$
\mathbb{E}\left(\exp \left(\eta\left\|\theta^{0}\left(t, \theta_{0}^{0}\right)\right\|^{2}\right)\left(1+\left\|\mathcal{J}_{0, t}\right\|\right)\right) \leq C_{1} \exp \left(\eta(1+4 R a \tilde{R} a) e^{-t / 2}\left\|\theta_{0}^{0}\right\|^{2}\right)
$$

where the operator $\mathcal{J}_{0, t}$ is the Fréchet derivative of $\theta^{0}\left(t, \theta_{0}\right)$ with respect to initial condition $\theta_{0}^{0}$; see (B.1) and (B.8) below.

(b) Gradient Bound for Markov semigroup: for any $\phi \in C_{b}^{1}(H)$, and every $t \geq 0, \theta \in H$

$$
\left\|\nabla P_{t}^{0} \phi(\theta)\right\| \leq C \exp \left(\eta\|\theta\|^{2}\right)\left(\sqrt{P_{t}^{0}\left(|\phi(\theta)|^{2}\right)}+\delta(t) \sqrt{P_{t}^{0}\left(\|\nabla \phi(\theta)\|^{2}\right)}\right),
$$

where $\delta(t) \rightarrow 0$ as $t \rightarrow \infty$. Here again $\delta:[0, \infty) \rightarrow[0, \infty)$ and $C>0$ depend only on Ra, $\tilde{R} a$, and $\eta$.

(c) Irreducibility condition: for any $M>0, \epsilon>0$ there is aF $t_{*}=t_{*}(M, \epsilon, \eta)$ such that for each $t \geq t_{*}$

$$
\inf _{\left\|\theta_{0}\right\|,\left\|\tilde{\theta}_{0}\right\| \leq M} \sup _{\Gamma \in \mathcal{C}\left(\delta_{\theta_{0}} P_{t}^{0}, \delta_{\tilde{\theta}_{0}} P_{t}^{0}\right)} \Gamma\left\{(\theta, \tilde{\theta}) \in H \times H: \rho_{\eta}(\theta, \tilde{\theta})<\epsilon\right\}>0
$$

where, as above in (2.19), $\mathcal{C}\left(\delta_{\theta_{0}} P_{t}^{0}, \delta_{\tilde{\theta}_{0}} P_{t}^{0}\right)$ denotes the collection of all couplings of the measures $\delta_{\theta_{0}} P_{t}^{0}$ and $\delta_{\tilde{\theta}_{0}} P_{t}^{0}$.

Proving the first item, (a), essentially reduces to establishing a moment bound which follows from estimates found in [22], and which we recall below in Appendix A (see Proposition A.2). The second condition, (4.2), can be translated to a control problem through the use of Malliavin calculus which in our setting amounts to proving a relatively straightforward Foias-Prodi type estimate. Once again (b) can be established by methods essentially contained in previous works and we relegate further details to Appendix B. As already mentioned above, the principal novel challenge here is to prove the irreducibility property (c) which we turn to next.

\subsection{Irreducibility}

In previous related works the proof of irreducibility essentially relies on the fact that the governing equations without the stochastic forcing have a trivial attractor which is stable under small force perturbations; see e.g. $[8,15,21,26]$. In our present situation, (2.4)-(2.5), the dynamics without body forces can be highly non-trivial. ${ }^{7}$ Here our approach reduces (4.3) to Foias-Prodi type estimates through the Girsanov theorem and careful stopping time arguments. We believe that our strategy may be applicable to other problems.

As a preliminary step we show that (4.3) follows from a simpler bound.

Lemma 4.1. For a given $N \geq 0$ consider (2.4)-(2.5) with $N$ independently forced directions. If for every $M, \epsilon>0$ there is a $t_{*}=t_{*}(M, \epsilon)>0$ such that

$$
\inf _{\left\|\theta_{0}\right\| \leq M} \mathbb{P}\left(\left\|\theta^{0}\left(t, \theta_{0}\right)\right\|<\epsilon\right)>0, \text { for each } t \geq t_{*},
$$

then (4.3) holds for such an $N$ and any $\eta>0$.

Proof. For any $\theta_{0}, \tilde{\theta}_{0} \in H$ consider the element $\tilde{\Gamma} \in \mathcal{C}\left(\delta_{\theta_{0}} P_{t}^{0}, \delta_{\tilde{\theta}_{0}} P_{t}^{0}\right)$ defined on cylindrical sets as

$$
\tilde{\Gamma}(A \times B)=P_{t}\left(\theta_{0}, A\right) \times P_{t}\left(\tilde{\theta}_{0}, B\right), \quad A, B \in \mathcal{B}(H) .
$$

\footnotetext{
${ }^{7}$ Note that the geometric control methods recently developed in [23] by the second coauthor would be difficult to apply, as these methods seemingly require a detailed understanding of the wave-number interactions in (2.4)-(2.5).
} 
For each $t>0$ and any $M, \eta, \gamma>0$ one has

$$
\begin{aligned}
\inf _{\left\|\theta_{0}\right\|,\left\|\tilde{\theta}_{0}\right\| \leq M} & \sup _{\Gamma \in \mathcal{C}\left(\delta_{\theta_{0}} P_{t}, \delta_{\tilde{\theta}_{0}} P_{t}\right)} \Gamma\left\{(\theta, \tilde{\theta}) \in H \times H: \rho_{\eta}(\theta, \tilde{\theta})<\gamma\right\} \\
& \geq \inf _{\left\|\theta_{0}\right\|,\left\|\tilde{\theta}_{0}\right\| \leq M} \tilde{\Gamma}\left\{(\theta, \tilde{\theta}) \in B_{1} \times B_{1}:\|\theta\|+\|\tilde{\theta}\|<\gamma \exp (-4 \eta)\right\} \\
& \geq\left(\inf _{\left\|\theta_{0}\right\| \leq M} P_{t}\left(\theta_{0},\{\theta \in H:\|\theta\|<\min \{\gamma / 2 \cdot \exp (-4 \eta), 1\}\}\right)\right)^{2} \\
& =\left(\inf _{\left\|\theta_{0}\right\| \leq M} \mathbb{P}\left(\left\|\theta\left(t, \theta_{0}\right)\right\|<\min \{\gamma / 2 \cdot \exp (-4 \eta), 1\}\right)\right)^{2},
\end{aligned}
$$

where we have used (2.23) in the first inequality. Applying (4.4) with $\epsilon=\min \{\gamma / 2 \cdot \exp (-4 \eta), 1\})$ and the given $M>0$ yields the desired result.

In order to establish (4.3) the rest of the section is therefore devoted to

Proposition 4.2. There exists $N_{0}=N_{0}(R a, \tilde{R a})$ sufficiently large (cf. (4.9)) such that, for any $N \geq N_{0}$ and every $M, \epsilon>0$, there is a $t_{*}=t_{*}(M, \epsilon)>0$ such that (4.4) is satisfied.

Proof of Proposition 4.2. We first establish the analogue of (4.4) for the modified system

$$
\begin{aligned}
& -\Delta \overline{\mathbf{u}}=\nabla \bar{p}+R a \hat{\mathbf{k}} \bar{\theta}, \quad \nabla \cdot \overline{\mathbf{u}}=0, \\
& d \bar{\theta}+\overline{\mathbf{u}} \cdot \nabla \bar{\theta} d t=\left(\tilde{R} a \cdot \bar{u}_{d}+\Delta \bar{\theta}-\lambda_{N} P_{N} \bar{\theta}\right) d t+\sum_{k=1}^{N} \sigma_{k} d W^{k}, \quad \bar{\theta}(0)=\theta_{0},
\end{aligned}
$$

when $N$ is sufficiently large. ${ }^{8}$ As in (2.1)-(2.2) we supplement (4.5)-(4.6) with the homogeneous boundary conditions (2.3). Denote $\psi:=\bar{\theta}-\sum_{k=1}^{N} \sigma_{k} W^{k}=\bar{\theta}-\sigma W$ which satisfies

$$
\partial_{t} \psi+\overline{\mathbf{u}} \cdot \nabla \psi=\tilde{R a} \cdot \bar{u}_{d}+\Delta \psi-\lambda_{N} P_{N} \psi+\left(\Delta \sigma W-\lambda_{N} P_{N} \sigma W-\overline{\mathbf{u}} \cdot \nabla(\sigma W)\right), \quad \psi(0)=\theta_{0} .
$$

Taking an inner product with $\psi$, using that $\overline{\mathbf{u}}$ is divergence free, the inverse Poincaré inequality (see (B.14) below) and the bound

$$
\|\nabla \overline{\mathbf{u}}\| \leq R a\|\bar{\theta}\| \leq R a(\|\psi\|+\|\sigma W\|)
$$

which follows from (4.5) we have

$$
\begin{aligned}
\frac{1}{2} \frac{d}{d t}\|\psi\|^{2}+\lambda_{N}\|\psi\|^{2} & \leq\left(\tilde{R} a\|\overline{\mathbf{u}}\|+\|\Delta \sigma W\|+\lambda_{N}\|\sigma W\|+\|\overline{\mathbf{u}}\|\|\nabla \sigma W\|_{L^{\infty}}\right)\|\psi\| \\
& \leq C\left(\tilde{R} a R a(\|\psi\|+\|\sigma W\|)+\|\Delta \sigma W\|+\lambda_{N}\|\sigma W\|+R a(\|\psi\|+\|\sigma W\|)\|\nabla \sigma W\|_{L^{\infty}}\right)\|\psi\| .
\end{aligned}
$$

For any $t>0$, let $\xi_{t}=\sup _{0<s<t}\{\|\psi(s)\|=0\}$ with the convection that the supremum of the empty set is zero. Thus, for any $t>0$, on the interval $\left[\xi_{t}, t\right]$ it follows that

$$
\frac{d}{d t}\|\psi\|+\left(\lambda_{N}-R a\left(\tilde{R} a+\|\nabla \sigma W\|_{L^{\infty}}\right)\right)\|\psi\| \leq\left(R a \tilde{R} a+\lambda_{N}+R a\|\nabla \sigma W\|_{L^{\infty}}\right)\|\sigma W\|+\|\Delta \sigma W\| .
$$

Next, we use the fact that, with positive probability, each of $\|\sigma W\|,\|\nabla \sigma W\|,\|\Delta \sigma W\|$ stays close to zero over finite time intervals. For $\gamma>0, t>0, N>0$ consider the sets

$$
\mathcal{X}_{\gamma, t, N}:=\left\{\sup _{s \in[0, t]}\|\nabla \sigma W\|_{L^{\infty}} \leq 1, \sup _{s \in[0, t]}\|\Delta \sigma W\| \leq \frac{\gamma}{2}, \sup _{s \in[0, t]}\|\sigma W\| \leq \gamma\left(\frac{1}{2\left(R a \tilde{R} a+\lambda_{N}+R a\right)} \wedge 1\right)\right\} .
$$

\footnotetext{
${ }^{8}$ Here recall that $P_{N}$ denotes the projection onto the first $N$ modes of $-\Delta$ (with boundary conditions as in $(2.3)$ ) and $\lambda_{N}$ is the corresponding largest eigenvalue in this collection.
} 
Since $\sigma$ is spatially smooth we infer from standard properties of Brownian motion that $\mathbb{P}\left(\mathcal{X}_{\gamma, t, N}\right)>0$ for any $\gamma>0, t>0$, and $N>0$. On the other hand, on $\mathcal{X}_{\gamma, t, N}$ the differential inequality

$$
\frac{d}{d t}\|\psi\|+\left(\lambda_{N}-R a(\tilde{R} a+1)\right)\|\psi\| \leq \gamma
$$

holds over the interval $\left[\xi_{t}, t\right]$.

Hence, fixing $N_{0}$ sufficiently large, we have for any $N \geq N_{0}$,

$$
\lambda_{N} \geq \max \{2 \operatorname{Ra}(\tilde{R} a+1), 1\},
$$

and we infer that on $\mathcal{X}_{\gamma, t, N}$,

$$
\left\|\bar{\theta}\left(t, \theta_{0}\right)\right\| \leq\|\psi(t)\|+\|\sigma W\| \leq 2 \gamma+e^{-\lambda_{N} t / 2}\left\|\theta_{0}\right\|,
$$

where note that $\|\psi(t)\|=0$ on the set where $\xi_{t}>0$. Therefore, for a given $M>0, \epsilon>0$, by choosing $\gamma=\epsilon / 4$ and $t_{*}=t_{*}(M, \epsilon)$ such that $e^{-\lambda_{N} t_{*}} M \leq \frac{\epsilon}{2}$, we have for any $t \geq t_{*}$

$$
\inf _{\left\|\theta_{0}\right\| \leq M} \mathbb{P}\left(\left\|\bar{\theta}\left(t, \theta_{0}\right)\right\|<\epsilon\right) \geq \mathbb{P}\left(\mathcal{X}_{\epsilon / 4, t, N}\right)>0 .
$$

In order to now infer (4.4) from (4.10) we apply the Girsanov theorem and make further bounds to a slightly modified version of (4.5)-(4.6). For $K>0$ and $\theta_{0} \in H$ define $\tilde{\theta}_{K}=\tilde{\theta}_{K}\left(\cdot, \theta_{0}\right)$ as the solution of (4.5)(4.6) with the term $-\lambda_{N} P_{N} \bar{\theta}$ replaced with $-\lambda_{N} P_{N} \tilde{\theta}_{K} \chi_{K}\left(\left\|P_{N} \tilde{\theta}_{K}\right\|\right)$. Here $\chi_{K}$ is a smooth, non-negative cut-off function with $\chi_{K} \equiv 1$ for $|x| \leq K$ and $\chi_{K} \equiv 0$ for $|x| \geq K+1$. Consider the stopping times

$$
\tau_{K}\left(\theta_{0}\right)=\inf _{s \geq 0}\left\{\left\|P_{N} \tilde{\theta}_{K}\left(s, \theta_{0}\right)\right\| \geq K\right\},
$$

for any $K>0$ and any $\theta_{0} \in H$. It is not hard to see that for any $K>0$ and any $\theta_{0} \in H$

$$
\mathbb{P}\left(\bar{\theta}\left(t \wedge \tau_{K}\left(\theta_{0}\right), \theta_{0}\right)=\tilde{\theta}_{K}\left(t \wedge \tau_{K}\left(\theta_{0}\right), \theta_{0}\right), \text { for every } t \geq 0\right)=1 .
$$

On the other hand, for any $\theta_{0} \in H$ and $K>0$, the law of $\tilde{\theta}_{K}\left(\cdot, \theta_{0}\right)$ is absolutely continuous with respect to the law of the processes $\theta^{0}\left(\cdot, \theta_{0}\right)$ solving (2.4)-(2.5). Indeed, for $\theta_{0} \in H$ and $K>0$ define

$$
\mathcal{M}_{\theta_{0}, K}(t)=\exp \left(-\int_{0}^{t} \alpha_{\theta_{0}, K} d W-\frac{1}{2} \int_{0}^{t}\left|\alpha_{\theta_{0}, K}\right|^{2} d s\right),
$$

where

$$
\alpha_{\theta_{0}, K}(s)=-\lambda_{N} \sigma^{-1} P_{N} \tilde{\theta}_{K}\left(s, \theta_{0}\right) \chi_{K}\left(\left\|P_{N} \tilde{\theta}_{K}\left(s, \theta_{0}\right)\right\|\right)
$$

and take

$$
d \mathbb{Q}_{\theta_{0}, K, t}:=\mathcal{M}_{\theta_{0}, K}(t) d \mathbb{P} .
$$

Notice that, since $\left|\sigma^{-1} P_{N} \tilde{\theta}_{K}\left(s, \theta_{0}\right) \chi_{K}\left(\left\|P_{N} \tilde{\theta}_{K}\right\|\right)\right| \leq\left\|\sigma^{-1}\right\| \cdot(K+1)$, the Novikov condition is satisfied and for any $\epsilon>0, t \geq 0, K>0$, and $\theta \in H$, the Girsanov theorem yields

$$
\mathbb{P}\left(\left\|\theta\left(t, \theta_{0}\right)\right\|<\epsilon\right)=\mathbb{Q}_{\theta_{0}, K, t}\left(\left\|\tilde{\theta}_{K}\left(t, \theta_{0}\right)\right\|<\epsilon\right)=\mathbb{E}\left(\mathcal{M}_{\theta_{0}, K}(t) \mathbb{1}_{\left\|\tilde{\theta}_{K}\left(t, \theta_{0}\right)\right\|<\epsilon}\right) .
$$

Hence, for any $\epsilon>0, \theta_{0} \in H$, and for any $], K, t>0$, the Markov inequality implies

$$
\mathbb{P}\left(\left\|\theta\left(t, \theta_{0}\right)\right\|<\epsilon\right) \geq \mathbb{I}\left(\left\|\tilde{\theta}_{K}\left(t, \theta_{0}\right)\right\|<\epsilon, \mathcal{M}_{\theta_{0}, K}(t) \geq \beth\right) \geq \mathbb{I}\left(\left\|\bar{\theta}\left(t, \theta_{0}\right)\right\|<\epsilon, \mathcal{M}_{\theta_{0}, K}(t) \geq \mathbb{J}, \tau_{K}\left(\theta_{0}\right)>t\right),
$$

where we used (4.11) for the final inequality. On the other hand

$$
\begin{aligned}
\mathbb{P}\left(\left\|\bar{\theta}\left(t, \theta_{0}\right)\right\|<\epsilon\right) & \leq \mathbb{P}\left(\left\|\bar{\theta}\left(t, \theta_{0}\right)\right\|<\epsilon, \mathcal{M}_{\theta_{0}, K}(t) \geq \beth\right)+\mathbb{P}\left(\mathcal{M}_{\theta_{0}, K}(t)<\beth\right) \\
& \leq \mathbb{P}\left(\left\|\bar{\theta}\left(t, \theta_{0}\right)\right\|<\epsilon, \mathcal{M}_{\theta_{0}, K}(t) \geq \beth, \tau_{K}\left(\theta_{0}\right)>t\right)+\mathbb{P}\left(\mathcal{M}_{\theta_{0}, K}(t)<\beth\right)+\mathbb{P}\left(\tau_{K}\left(\theta_{0}\right)<t\right) .
\end{aligned}
$$


These two bounds yield

$$
\begin{aligned}
\frac{1}{\bar{J}} \inf _{\left\|\theta_{0}\right\| \leq M} \mathbb{P}\left(\left\|\theta\left(t, \theta_{0}\right)\right\|<\epsilon\right) \\
\quad \inf _{\left\|\theta_{0}\right\| \leq M} \mathbb{P}\left(\left\|\bar{\theta}\left(t, \theta_{0}\right)\right\|<\epsilon\right)-\sup _{\left\|\theta_{0}\right\| \leq M}\left(\mathbb{P}\left(\mathcal{M}_{\theta_{0}, K}(t)<\boldsymbol{I}\right)+\mathbb{P}\left(\tau_{K}\left(\theta_{0}\right)<t\right)\right),
\end{aligned}
$$

for any $M, \epsilon, t>0$ and for any $K, \beth>0$.

Since the first term on the the right-hand side of (4.13) is independent of $K>0$ and $I>0$, we finish the argument by showing that for every fixed $M, K, t>0$

$$
\sup _{\left\|\theta_{0}\right\| \leq M} \mathbb{P}\left(\mathcal{M}_{\theta_{0}, K}(t)<\boldsymbol{I}\right) \rightarrow 0, \quad \text { as } \boldsymbol{I} \rightarrow 0,
$$

and for every given $M, t>0$

$$
\sup _{\left\|\theta_{0}\right\| \leq M} \mathbb{P}\left(\tau_{K}\left(\theta_{0}\right)<t\right) \rightarrow 0, \quad \text { as } K \rightarrow \infty .
$$

For the first bound (4.14), we have from (4.12) and Itō isometry

$$
\begin{aligned}
\mathbb{P}\left(\mathcal{M}_{\theta_{0}, K}(t)<\boldsymbol{I}\right) & =\mathbb{P}\left(\int_{0}^{t} \alpha_{\theta_{0}, K} d W+\frac{1}{2} \int_{0}^{t}\left|\alpha_{\theta_{0}, K}\right|^{2} d s>\log \left(\beth^{-1}\right)\right) \\
& \leq \frac{1}{\log \left(\beth^{-1}\right)} \mathbb{E}\left(\left|\int_{0}^{t} \alpha_{\theta_{0}, K} d W\right|+\frac{1}{2} \int_{0}^{t}\left|\alpha_{\theta_{0}, K}\right|^{2} d s\right) \\
& \leq \frac{2}{\log \left(\beth^{-1}\right)} \mathbb{E}\left(1+\lambda_{N}^{2}\left\|\sigma^{-1} P_{N}\right\|^{2} \int_{0}^{t}\left\|P_{N} \tilde{\theta}\left(t, \theta_{0}\right)\right\|^{2} \chi_{K}\left(\left\|P_{N} \tilde{\theta}_{K}\left(t, \theta_{0}\right)\right\|\right) d s\right), \\
& \leq \frac{2\left(1+\lambda_{N}^{2}\left\|\sigma^{-1} P_{N}\right\|^{2}(K+1)^{2} t\right)}{\log \left(\beth^{-1}\right)},
\end{aligned}
$$

valid for any $I \in(0,1), K>0$, and any $\theta_{0} \in H$. For the second bound, (4.15) observe that, in view of (4.11),

$$
\mathbb{P}\left(\tau_{K}\left(\theta_{0}\right)<t\right) \leq \mathbb{P}\left(\sup _{s \in[0, t]}\left\|P_{N} \bar{\theta}\left(s, \theta_{0}\right)\right\| \geq K\right) \leq \frac{1}{K^{2}} \mathbb{E}\left(\sup _{s \in[0, t]}\left\|\bar{\theta}\left(s, \theta_{0}\right)\right\|^{2}\right) .
$$

From the Itō formula, and (1.4), it follows that

$$
d\|\bar{\theta}\|^{2}+2 \lambda_{N}\left\|P_{N} \bar{\theta}\right\|^{2} d t+2\|\nabla \bar{\theta}\|^{2} d t=\left(2 \tilde{R} a\left\langle\tilde{u}_{d}, \bar{\theta}\right\rangle+1\right) d t+\langle\sigma, \bar{\theta}\rangle d W .
$$

Integrating in time and using (4.5), inverse Poincaré inequality (see (B.14)), and (4.7) we infer for any $s \geq 0$

$$
\|\bar{\theta}(s)\|^{2}+2 \lambda_{N} \int_{0}^{s}\|\bar{\theta}\|^{2} d r \leq\left\|\theta_{0}\right\|^{2}+2 R a \tilde{R} a \int_{0}^{s}\|\bar{\theta}\|^{2} d r+s+2 \sup _{r \in[0, s]}\left|\int_{0}^{r}\langle\sigma, \bar{\theta}\rangle d W\right| .
$$

Using the assumption (4.9) and the Birkholder-Davis-Gundy inequality we infer

$$
\mathbb{E}\left(\sup _{s \in[0, t]}\left\|\bar{\theta}\left(s, \theta_{0}\right)\right\|^{2}\right) \leq\left\|\theta_{0}\right\|^{2}+17 t .
$$

Combining (4.16) and (4.17) thus yields the second bound (4.15).

Using (4.10), (4.13), (4.14), and (4.15), we conclude the proof as follows. Given any $\epsilon>0$ and any $M>0$, and with $\lambda_{N}$ given as in (4.9), choose $t_{*}$ as in (4.10), that is, such that $e^{-\lambda_{N} t_{*}} M \leq \frac{\epsilon}{2}$. Fix any $t \geq t_{*}$ and by (4.10) we have $a=a(M, \epsilon, t):=\inf _{\left\|\theta_{0}\right\| \leq M} \mathbb{P}\left(\left\|\bar{\theta}\left(t, \theta_{0}\right)\right\|<\epsilon\right)>0$. Now, by (4.15), we can pick $K$ sufficiently large so that $\sup _{\left\|\theta_{0}\right\| \leq M} \mathbb{P}\left(\tau_{K}\left(\theta_{0}\right)<t\right) \leq a / 4$, and with $K, M, t$ fixed, we can by (4.14) choose I $>0$ small enough so that $\sup _{\left\|\theta_{0}\right\| \leq M} \mathbb{P}\left(\mathcal{M}_{K, \theta_{0}}(t)<\boldsymbol{I}\right) \leq a / 4$. Finally, by combining these choices with (4.13) we obtain that

$$
\inf _{\left\|\theta_{0}\right\| \leq M} \mathbb{P}\left(\left\|\theta\left(t, \theta_{0}\right)\right\|<\epsilon\right) \geq \frac{\Xi a}{2}>0 .
$$

The proof of Proposition 4.2 is thus complete. 


\section{Finite Time Asymptotics}

In this section we prove Theorem 2.1. We also derive a 'corrector' system which we show approximates the velocity component of the full system (2.1)-(2.2) up to the initial time (see Theorem 5.1 below).

\subsection{Preliminaries: The Stokes Operator}

Before proceeding further we recall (see e.g. $[7,40]$ ) some properties of solutions of the Stokes equation

$$
-\Delta \mathbf{u}=\nabla p+\mathbf{f}, \quad \nabla \cdot \mathbf{u}=0
$$

supplemented with the mixed periodic-Dirichlet boundary conditions as in (2.3). We can express (5.1) more abstractly as $A \mathbf{u}=P \mathbf{f}$, where $A=-P \Delta$ is the Stokes operator. Here, $P$ is the Leray projection on divergence free vector fields $P:\left(L^{2}(\mathcal{D})\right)^{3} \rightarrow H_{1}$ with $H_{1}$, the space of $L^{2}$ divergence free vector fields, defined in Section 2.1. ${ }^{9}$ As in the classical elliptic theory we have that for any $\mathbf{f} \in\left(L^{2}(\mathcal{D})\right)^{3}$, there exists a unique $\mathbf{u} \in D(A)=V_{1} \cap\left(H^{2}(\mathcal{D})\right)^{3}$ which satisfies

$$
\|\mathbf{u}\|_{H^{2}} \leq C\|\mathbf{f}\|
$$

where $C$ is independent of $\mathbf{f}$. In what follows we frequently denote $\mathbf{u}=A^{-1} P \mathbf{f}$.

Since $A$ is a positive, self-adjoint operator which is unbounded on the space $H_{1}$ with a compact inverse, by Hilbert's theorem there is a complete orthonormal basis of eigenfunctions $\left\{\mathbf{e}_{k}\right\}_{k \geq 1}$ of $A$ with the associated non-decreasing sequence of eigenvalues $\lambda_{k}^{*}$ diverging to infinity. Take

$$
\mathcal{P}_{N} \text { to be the projection onto the subspace } H_{N}:=\operatorname{span}\left\{\mathbf{e}_{1}, \ldots, \mathbf{e}_{N}\right\} \text {. }
$$

Here the regularity theory as found in, say [7, 40], show that each $\mathbf{e}_{k}$ is smooth and hence in particular $H_{N} \subset V$.

We also consider the associated linear evolution given as

$$
\partial_{t} \mathbf{u}-\mu \Delta \mathbf{u}=\nabla p+\mathbf{f}, \quad \nabla \cdot \mathbf{u}=0, \quad \mathbf{u}(0)=\mathbf{u}_{0},
$$

for any parameter $\mu>0$ and relative to the (sufficiently regular) data $\mathbf{f}, \mathbf{u}_{0}$ supplemented with the boundary conditions (2.3). Here, for any $\mathbf{f} \in L_{l o c}^{2}\left([0, \infty) ; H_{1}\right)$ and $\mathbf{u}_{0} \in H_{1}$ there exists a unique solution $\mathbf{u}$ of (5.4) with $\mathbf{u} \in L_{l o c}^{2}\left([0, \infty) ; V_{1}\right) \cap C\left([0, \infty) ; H_{1}\right)$. Moreover, $A$ is the generator of an analytic semigroup which we denote as $\{\exp (-\mu A t)\}_{t \geq 0}$.

\subsection{Finite Time Convergence Estimates}

We next turn to the proof of Theorem 2.1:

Proof of Theorem 2.1. Take $\phi^{\varepsilon}=\theta^{\varepsilon}-\theta^{0}$ and $\mathbf{v}^{\varepsilon}=\mathbf{u}^{\varepsilon}-\mathbf{u}^{0}$ with $\mathbf{u}^{0}=M\left(\theta^{0}\right)$, where $M$ is defined by (2.9). Referring to (2.1)-(2.3) and (2.4)-(2.5) we see that $\phi^{\varepsilon}$ satisfies

$$
\partial_{t} \phi^{\varepsilon}-\Delta \phi^{\varepsilon}=\tilde{R} a \cdot v_{3}^{\varepsilon}-\mathbf{v}^{\varepsilon} \cdot \nabla \theta^{0}-\mathbf{u}^{\varepsilon} \cdot \nabla \phi^{\varepsilon}, \quad \phi^{\varepsilon}(0)=\theta^{\varepsilon}(0)-\theta^{0}(0):=\phi_{0}^{\varepsilon} .
$$

Therefore, taking an $L^{2}$ inner product with $\phi^{\varepsilon}$ and using that $\nabla \cdot \mathbf{v}^{\varepsilon}=0$ we have

$$
\frac{1}{2} \frac{d}{d t}\left\|\phi^{\varepsilon}\right\|^{2}+\left\|\nabla \phi^{\varepsilon}\right\|^{2}=\int\left(\tilde{R} a \cdot v_{d}^{\varepsilon}-\mathbf{v}^{\varepsilon} \cdot \nabla \theta^{0}\right) \phi^{\varepsilon} d x \leq \tilde{R} a\left\|\mathbf{v}^{\varepsilon}\right\|\left\|\phi^{\varepsilon}\right\|+\left\|\mathbf{v}^{\varepsilon}\right\|_{L^{6}}\left\|\nabla \phi^{\varepsilon}\right\|\left\|\theta^{0}\right\|_{L^{3}} .
$$

Hence from standard Sobolev embeddings, Young's inequality, and the Poincaré inequality we obtain

$$
\frac{d}{d t}\left\|\phi^{\varepsilon}\right\|^{2} \leq C\left(\left\|\theta^{0}\right\|_{L^{3}}^{2}+\tilde{R} a^{2}\right)\left\|\nabla \mathbf{v}^{\varepsilon}\right\|^{2}
$$

\footnotetext{
${ }^{9}$ Equivalently $A \mathbf{u}=-\Delta \mathbf{u}-\nabla p$, where $p=p(\mathbf{u})$ the 'pressure' is the unique $H^{1}$ function satisfying $\Delta p=-\operatorname{div}(\Delta u)$ in the weak sense.
} 
Integrating in time we infer that

$$
\sup _{s \in[0, t]}\left\|\phi^{\varepsilon}(s \wedge \tau)\right\|^{2} \leq\left\|\phi_{0}^{\varepsilon}\right\|^{2}+\sup _{s \in[0, t \wedge \tau]}\left(\left\|\theta^{0}(s)\right\|_{L^{3}}^{2}+\tilde{R a^{2}}\right) \int_{0}^{t \wedge \tau}\left\|\nabla \mathbf{v}^{\varepsilon}\left(t^{\prime}\right)\right\|^{2} d t^{\prime}
$$

for any $t>0$ and any stopping time $\tau \geq 0$.

We now turn to derive an evolution equation for $\mathbf{v}^{\varepsilon}$. Recalling that $\mathbf{u}^{\varepsilon}$ and $\mathbf{u}^{0}$ satisfy respectively, (2.1) and (2.4) we find

$$
\varepsilon\left(\partial_{t} \mathbf{u}^{\varepsilon}+\mathbf{u}^{\varepsilon} \cdot \nabla \mathbf{u}^{\varepsilon}\right)-\Delta \mathbf{v}^{\varepsilon}=\nabla q^{\varepsilon}+R a \hat{\mathbf{k}} \phi^{\varepsilon},
$$

where $q^{\varepsilon}=\tilde{p}^{\varepsilon}-\tilde{p}$ is the difference in the pressures. On the other hand, recalling that $\mathbf{u}^{0}=R a A^{-1}\left(P\left(\hat{\mathbf{k}} \theta^{0}\right)\right)$, we have

$$
d \mathbf{u}^{0}=-R a A^{-1} P\left(\hat{\mathbf{k}}\left(\mathbf{u}^{0} \cdot \nabla \theta^{0}-\Delta \theta^{0}-\tilde{R} a \cdot u_{3}^{0}\right)\right) d t+R a \sum_{k=1}^{N} A^{-1} P\left(\hat{\mathbf{k}} \sigma_{k}\right) d W^{k} .
$$

Multiplying (5.6) by $\varepsilon^{-1}$, subtracting the resulting system from (5.7) and rearranging we obtain

$$
\begin{aligned}
d \mathbf{v}^{\varepsilon}- & \frac{1}{\varepsilon} \Delta \mathbf{v}^{\varepsilon} d t=\frac{1}{\varepsilon}\left(\nabla q^{\varepsilon}+R a \hat{\mathbf{k}} \phi^{\varepsilon}\right) d t \\
& +\left(R a A^{-1} P\left(\hat{\mathbf{k}}\left(\mathbf{u}^{0} \cdot \nabla \theta^{0}-\Delta \theta^{0}+\tilde{R} a \cdot u_{d}^{0}\right)\right)-\mathbf{u}^{\varepsilon} \cdot \nabla \mathbf{u}^{\varepsilon}\right) d t-R a \sum_{k=1}^{N} A^{-1} P\left(\hat{\mathbf{k}} \sigma_{k}\right) d W^{k},
\end{aligned}
$$

with $\nabla \cdot \mathbf{v}^{\varepsilon}=0$.

Using (5.8) we estimate $\mathbf{v}^{\varepsilon}$ as follows. The Itō formula and (5.9) yields

$$
\begin{aligned}
d\left\|\mathbf{v}^{\varepsilon}\right\|^{2}+\frac{2}{\varepsilon}\left\|\nabla \mathbf{v}^{\varepsilon}\right\|^{2} d t= & \frac{2}{\varepsilon} R a\left\langle\phi^{\varepsilon}, v_{d}^{\varepsilon}\right\rangle d t+2\left\langle R a A^{-1} P\left(\hat{\mathbf{k}}\left(\mathbf{u}^{0} \cdot \nabla \theta^{0}-\Delta \theta^{0}-\tilde{R} a \cdot u_{d}^{0}\right)\right)-\mathbf{u}^{\varepsilon} \cdot \nabla \mathbf{u}^{\varepsilon}, \mathbf{v}^{\varepsilon}\right\rangle d t \\
& +R a^{2} \sum_{k=1}^{N}\left|A^{-1} P\left(\hat{\mathbf{k}} \sigma_{k}\right)\right|^{2} d t-2 R a \sum_{k=1}^{N}\left\langle A^{-1} P\left(\hat{\mathbf{k}} \sigma_{k}\right), \mathbf{v}^{\varepsilon}\right\rangle d W^{k} \\
:= & \left(T_{1}+T_{2}+T_{3}+T_{4}+T_{5}+T_{6}\right) d t+S d W .
\end{aligned}
$$

With the Young and Poincaré inequalities we have

$$
\left|T_{1}\right| \leq \frac{1}{4 \varepsilon}\left\|\nabla \mathbf{v}^{\varepsilon}\right\|^{2}+\frac{4 R a^{2}}{\varepsilon}\left\|\phi^{\varepsilon}\right\|^{2} .
$$

For $T_{2}$ we use that $A^{-1}$ is self-adjoint on $H, D(A) \subset H$ and that $\mathbf{u}^{0}, \mathbf{v}^{\varepsilon}$ are divergence free to obtain

$$
\left|T_{2}\right|=2 R a\left|\int \mathbf{u}^{0} \cdot \nabla \theta^{0}\left(A^{-1} \mathbf{v}^{\varepsilon}\right)_{3} d x\right|=2 R a\left|\int \mathbf{u}^{0} \cdot \nabla\left(A^{-1} \mathbf{v}^{\varepsilon}\right)_{3} \theta^{0} d x\right|,
$$

where $\left(A^{-1} \mathbf{v}^{\varepsilon}\right)_{3}$ represents the third component of the vector field $A^{-1} \mathbf{v}^{\varepsilon}$. Hence (5.2) and the imbedding $H^{2} \hookrightarrow L^{\infty}$ imply

$$
\left|T_{2}\right| \leq 2 R a\left\|\mathbf{u}^{0}\right\|\left\|\theta^{0}\right\|\left\|\nabla\left(A^{-1} \mathbf{v}^{\varepsilon}\right)\right\|_{L^{\infty}} \leq C R a^{2}\left\|\theta^{0}\right\|^{2}\left\|\nabla \mathbf{v}^{\varepsilon}\right\| \leq \frac{1}{4 \varepsilon}\left\|\nabla \mathbf{v}^{\varepsilon}\right\|^{2}+\varepsilon C R a^{4}\left\|\theta^{0}\right\|^{4} .
$$

For the terms $T_{3}$ and $T_{4}$ we use the regularity of the Stokes operator to obtain

$$
\begin{aligned}
\left|T_{3}\right|+\left|T_{4}\right| & \leq \frac{1}{4 \varepsilon}\left\|\nabla \mathbf{v}^{\varepsilon}\right\|^{2}+4 \varepsilon R a^{2}\left(\left\|\theta^{0}\right\|^{2}+\tilde{R a^{2}}\left\|\mathbf{u}^{0}\right\|^{2}\right) \\
& \leq \frac{1}{4 \varepsilon}\left\|\nabla \mathbf{v}^{\varepsilon}\right\|^{2}+\varepsilon R a^{2}\left(\tilde{R a^{2}} R a^{2}+1\right) C\left\|\theta^{0}\right\|^{2} .
\end{aligned}
$$


The most delicate term is $T_{5}$. Here we take advantage of an additional cancellation to obtain extra regularity. Since $\mathbf{u}^{\varepsilon}=\mathbf{v}^{\varepsilon}+\mathbf{u}^{0}$ we find

$$
\begin{aligned}
\left|T_{5}\right| & =2\left|\left\langle\mathbf{u}^{\varepsilon} \cdot \nabla \mathbf{u}^{0}, \mathbf{v}^{\varepsilon}\right\rangle\right|=2\left|\left\langle\mathbf{u}^{\varepsilon} \cdot \nabla \mathbf{v}^{\varepsilon}, \mathbf{u}^{0}\right\rangle\right| \leq \frac{1}{4 \varepsilon}\left\|\nabla \mathbf{v}^{\varepsilon}\right\|^{2}+4 \varepsilon\left\|\mathbf{u}^{0}\right\|_{L^{\infty}}^{2}\left\|\mathbf{u}^{\varepsilon}\right\|^{2} \\
& \leq \frac{1}{4 \varepsilon}\left\|\nabla \mathbf{v}^{\varepsilon}\right\|^{2}+\varepsilon C R a^{2}\left(\left\|\theta^{0}\right\|^{4}+\left\|\mathbf{u}^{\varepsilon}\right\|^{4}\right),
\end{aligned}
$$

where we used the imbedding $H^{2} \hookrightarrow L^{\infty}$ and (5.2) for the final bound. Finally we observe $\left|T_{6}\right| \leq C R a^{2}$.

Combining the bounds (5.10)-(5.13) and rearranging in (5.9) we find

$$
\begin{aligned}
d\left\|\mathbf{v}^{\varepsilon}\right\|^{2}+\frac{1}{\varepsilon}\left\|\nabla \mathbf{v}^{\varepsilon}\right\|^{2} d t \leq & \frac{4 R a^{2}}{\varepsilon}\left\|\phi^{\varepsilon}\right\|^{2} d t+\varepsilon C\left(1+R a^{4}\right)\left(1+\tilde{R} a^{2}\right)\left(\left\|\theta^{0}\right\|^{4}+\left\|\mathbf{u}^{\varepsilon}\right\|^{4}+1\right) d t \\
& +C R a^{2} d t+2 R a \sum_{k=1}^{N}\left\langle A^{-1} P\left(\hat{\mathbf{k}} \sigma_{k}\right), \mathbf{v}^{\varepsilon}\right\rangle d W^{k}
\end{aligned}
$$

where the constant $C>0$ is independent of $R a, \tilde{R} a$, and $\varepsilon>0$. Consequently, for any $t \geq 0$ and any stopping time $\tau$, we have

$$
\begin{aligned}
\int_{0}^{t \wedge \tau}\left\|\nabla \mathbf{v}^{\varepsilon}\right\|^{2} d t \leq & \varepsilon\left\|\mathbf{v}^{\varepsilon}(0)\right\|^{2}+4 R a^{2} \int_{0}^{t \wedge \tau}\left(\left\|\phi^{\varepsilon}\right\|^{2}+\varepsilon C\right) d t^{\prime}+\varepsilon^{2} C\left(1+R a^{4}\right)\left(1+\tilde{R} a^{2}\right) \int_{0}^{t \wedge \tau}\left(\left\|\theta^{0}\right\|^{4}+\left\|\mathbf{u}^{\varepsilon}\right\|^{4}+1\right) d t^{\prime} \\
& -\varepsilon R a \sum_{k=1}^{N} \int_{0}^{t \wedge \tau}\left\langle A^{-1} P\left(\hat{\mathbf{k}} \sigma_{k}\right), \mathbf{v}^{\varepsilon}\right\rangle d W^{k}
\end{aligned}
$$

where $C$ is independent of $\varepsilon>0, R a, \tilde{R} a$, and $\tau$.

Next for any $\kappa>0$ define the stopping times

$$
\tau_{\kappa}:=\inf _{t \geq 0}\left\{\left\|\theta^{0}(t)\right\|_{L^{3}}^{2} \geq \kappa\right\}
$$

From this definition and the bounds (5.5), (5.15) we now infer

$$
\begin{aligned}
\mathbb{E} \sup _{s \in[0, t]}\left\|\phi^{\varepsilon}\left(s \wedge \tau_{\kappa}\right)\right\|^{2} \\
\leq \mathbb{E}\left\|\phi_{0}^{\varepsilon}\right\|^{2}+4 R a^{2}\left(\kappa+\tilde{R} a^{2}\right) \int_{0}^{t} \mathbb{E}\left(\sup _{s \in\left[0, t^{\prime}\right]}\left\|\phi^{\varepsilon}\left(s \wedge \tau_{\kappa}\right)\right\|^{2}\right) d t^{\prime}+\varepsilon\left(\kappa+\tilde{R} a^{2}\right)\left(\mathbb{E}\left\|\mathbf{v}^{\varepsilon}(0)\right\|^{2}+R a^{2} C t\right) \\
\quad+\varepsilon^{2} C\left(\kappa+\tilde{R} a^{4}+1\right)\left(\operatorname{Ra}^{4}+1\right) \int_{0}^{t} \mathbb{E}\left(\left\|\theta^{0}\right\|^{4}+\left\|\mathbf{u}^{\varepsilon}\right\|^{4}+1\right) d t^{\prime}
\end{aligned}
$$

which implies with the Gronwall inequality that

$$
\mathbb{E} \sup _{s \in[0, t]}\left\|\phi^{\varepsilon}\left(s \wedge \tau_{\kappa}\right)\right\|^{2} \leq \exp \left(C\left(R a^{4}+1\right)\left(\kappa+\tilde{R} a^{4}+1\right)(t+1)\right)\left(\varepsilon \mathcal{M}_{\varepsilon}(t)+\mathbb{E}\left\|\phi_{0}^{\varepsilon}\right\|^{2}\right)
$$

where

$$
\mathcal{M}_{\varepsilon}(t):=\mathbb{E}\left\|\mathbf{v}^{\varepsilon}(0)\right\|^{2}+\int_{0}^{t}\left[\varepsilon \mathbb{E}\left(\left\|\mathbf{u}^{\varepsilon}\right\|^{4}+\left\|\theta^{0}\right\|^{4}\right)+1\right] d t^{\prime}
$$

and the constant $C$ is independent of $\kappa, \varepsilon, R a, \tilde{R} a$, and $t$. By (2.26) and our standing assumption that (2.12) holds, we observe that $\mathcal{M}_{\varepsilon}$ is bounded independently of $\varepsilon>0$ and we obtain

$$
\mathbb{E}\left(\sup _{s \in[0, t]}\left\|\phi^{\varepsilon}(s)\right\|^{2} \mathbb{1}_{\tau_{\kappa}>t}\right) \leq \mathbb{E} \sup _{s \in[0, t]}\left\|\phi^{\varepsilon}\left(s \wedge \tau_{\kappa}\right)\right\|^{2} \leq C_{1}\left(\varepsilon+\mathbb{E}\left\|\phi_{0}^{\varepsilon}\right\|^{2}\right) \exp \left(C_{1} \kappa\right)
$$


where the constant $C_{1}=C(R a, \tilde{R} a, t)$ is independent of $\varepsilon>0$ and $\kappa>0$.

Set $X_{\varepsilon}(t):=\sup _{s \in[0, t]}\left\|\phi^{\varepsilon}(s)\right\|^{2}$ and for each $t \geq 0, \kappa>0, \varepsilon>0$ define the sets

$$
E_{t, \kappa, \varepsilon}:=\left\{\sup _{s \in[0, t]}\left\|\theta^{0}(s)\right\|_{L^{3}}^{2} \geq \kappa\right\}=\left\{\tau_{\kappa} \leq t\right\} .
$$

For each $t>0$ one finds by the Markov inequality, Proposition A.1, and the assumption (2.26) that for sufficiently small $\eta_{1}=\eta_{1}(\tilde{R} a, \eta)>0$,

$$
\mathbb{P}\left(E_{t, \kappa, \varepsilon}\right) \leq e^{-\eta \kappa} \mathbb{E} \exp \left(\eta_{1} \sup _{s \in[0, t]}\left\|\theta^{0}(s)\right\|_{L^{3}}^{2}\right) \leq C_{2} e^{-\eta \kappa},
$$

where $C_{2}=C_{2}\left(C_{0}, R a, \tilde{R} a,\|\sigma\|_{L^{3}}, \eta, t\right)>0$ is independent of $\varepsilon>0$ and $\kappa>0$. On the other hand, for any $\gamma \in(0,1)$ we have

$$
\begin{aligned}
\mathbb{E} X_{\varepsilon}(t)^{\gamma} & \left.=\sum_{k=0}^{\infty} \mathbb{E}\left(X_{\varepsilon}(t)^{\gamma} \mathbb{1}_{k \leq\left(\sup _{s \in[0, t]}\left\|\theta^{0}(s)\right\|_{L^{3}}^{2}\right.}\right)<k+1\right)=\sum_{k=0}^{\infty} \mathbb{E}\left(X_{\varepsilon}(t)^{\gamma} \mathbb{1}_{\tau_{k} \leq t} \mathbb{1}_{\tau_{k+1}>t}\right) \\
& \leq \sum_{k=0}^{\infty}\left(\mathbb{E}\left(X_{\varepsilon}(t) \mathbb{1}_{\tau_{k+1}>t}\right)\right)^{\gamma}\left(\mathbb{P}\left(E_{t, \kappa, \varepsilon}\right)\right)^{1-\gamma} \\
& \leq C\left(\varepsilon+\mathbb{E}\left\|\phi_{0}^{\varepsilon}\right\|^{2}\right)^{\gamma} \sum_{k=0}^{\infty} \exp \left(\gamma C_{1}(k+1)-(1-\gamma) \eta k\right),
\end{aligned}
$$

where we have used (5.18) and (5.19) for the final bound. Here, $C=C\left(C_{0}, R a, \tilde{R} a,\|\sigma\|_{L^{3}}, \eta, t\right)$ is independent of $\varepsilon>0, \mathbb{E}\left\|\phi_{0}^{\varepsilon}\right\|^{2}$ and $C_{1}$ is the constant appearing in (5.18). Thus when $\gamma<\frac{\eta}{C_{1}+\eta}$ the series in (5.20) converges. Then for any $p>0$ and any $\gamma<\left(\frac{\eta}{C_{1}+\eta}\right) \wedge p$ we find

$$
\mathbb{E} \sup _{s \in[0, t]}\left\|\phi^{\varepsilon}(s)\right\|^{p} \leq C\left(\mathbb{E} \sup _{s \in[0, t]}\left(\left\|\theta^{\varepsilon}(s)\right\|^{2(p-\gamma)}+\left\|\theta^{0}(s)\right\|^{2(p-\gamma)}\right)\right)^{1 / 2}\left(\mathbb{E} \sup _{s \in[0, t]}\left\|\phi^{\varepsilon}(s)\right\|^{2 \gamma}\right)^{1 / 2} .
$$

Combing this bound with (2.26) and (5.20) we now obtain the first part of (2.27).

To address the second term in (2.27) we return to (5.15). Taking expected values we obtain

$$
\mathbb{E} \int_{0}^{t}\left\|\nabla \mathbf{v}^{\varepsilon}\right\|^{2} d t \leq \varepsilon\left(\mathbb{E}\left\|\mathbf{v}^{\varepsilon}(0)\right\|^{2}+C\right)+C \mathbb{E}\left(\sup _{s \in[0, t]}\left\|\phi^{\varepsilon}(s)\right\|^{2}\right)
$$

where $C=C\left(R a, \tilde{R} a, t, C_{0}\right)$ is independent of $\varepsilon>0$. Combining this observation with the previous bound, the proof of Theorem 2.1 is now complete.

\subsection{Approximation up to Initial Conditions: The Corrector}

We next formally derive and then rigorously analyze a refined approximation of (2.1)-(2.2). By Theorem 2.1, the velocity component $M\left(\theta^{0}\right)$ of the limit system (2.4)-(2.5) well approximates the velocity field $\mathbf{u}^{\varepsilon}$ of the full system $(2.1)-(2.2)$ in the norm $L^{2}\left([0, t], H^{1}(\mathcal{D})\right)$ for each fixed $t>0$. Also Theorem 2.2, (ii) shows that the invariant measure of the limit system approximates any invariant state of the full system, which can be interpreted as a approximation of laws of solutions as $t \rightarrow \infty$. On the other hand, we do not expect (2.4)-(2.5) to accurately describe the behavior of (2.1)-(2.2) up to $t=0$ due the presence of a (initial time) boundary layer.

We next derive the so called 'corrector equation' which provides effective dynamics for (2.1)-(2.2) and which is globally well-posed and whose velocity component remains close to the dynamics of (2.1)-(2.2) in $L^{\infty}\left([0, T], L^{2}(\mathcal{D})\right)$, that is, even up to time zero. Note that similar considerations motivate the analysis in [44] which treats such small time approximations in the deterministic setting. 


\section{Formal Derivation}

In order to identify multiple time scales in $(2.1)-(2.2)$ we introduce an additional 'slow time' variable $\varsigma=\varepsilon t$. We then replace

$$
\partial_{t} \rightarrow \partial_{t}+\frac{1}{\varepsilon} \partial_{\varsigma}
$$

Under this ansatz the momentum equation (2.1) becomes

$$
\varepsilon\left(\partial_{t} \mathbf{u}^{\varepsilon}+\mathbf{u}^{\varepsilon} \cdot \nabla \mathbf{u}^{\varepsilon}\right)+\partial_{\varsigma} \mathbf{u}^{\varepsilon}-\Delta \mathbf{u}^{\varepsilon}=\nabla \tilde{p}^{\varepsilon}+R a \hat{\mathbf{k}} \theta^{\varepsilon}
$$

Dropping the terms of order $\varepsilon$ and using Duhamel's formula we obtain

$$
\mathbf{u}^{\varepsilon}(\varsigma)=e^{-A \varsigma} \mathbf{u}^{\varepsilon}(0)+\int_{0}^{\varsigma} e^{-A(\varsigma-r)} P\left(\operatorname{Ra} \hat{\mathbf{k}} \theta^{\varepsilon}\right) d r
$$

where as in Section 5.1, $e^{-A \varsigma}$ denotes the semigroup whose generator is the Stokes operator $A$.

The form of (2.1) suggests that $\mathbf{u}^{\varepsilon}$ fluctuates rapidly in comparison to $\theta^{\varepsilon}$. Under the further ansatz that there is a clear separation of time scales between the motion of $\mathbf{u}^{\varepsilon}$ and that of $\theta^{\varepsilon}$ we suppose that $\theta^{\varepsilon}$ is independent of $\varsigma$. From (5.21) this yields

$$
\begin{aligned}
\mathbf{u}^{\varepsilon}(\varsigma) & =e^{-A \varsigma} \mathbf{u}^{\varepsilon}(0)+A^{-1}\left(P\left(R a \cdot \hat{\mathbf{k}} \tilde{\theta}^{\varepsilon}\right)\right)-e^{-A_{\varsigma}} A^{-1}\left(P\left(R a \hat{k} \theta^{\varepsilon}\right)\right) \\
& :=A^{-1}\left(P\left(R a \cdot \hat{\mathbf{k}} \tilde{\theta}^{\varepsilon}\right)\right)+\mathbf{w}^{\varepsilon}(\varsigma)
\end{aligned}
$$

where $\mathbf{w}^{\varepsilon}$ solves

$$
\partial_{\varsigma} \mathbf{w}^{\varepsilon}-\Delta \mathbf{w}^{\varepsilon}=\nabla q^{\varepsilon}, \quad \mathbf{w}^{\varepsilon}(0)=\mathbf{u}^{\varepsilon}(0)-\mathbf{y}^{\varepsilon} \quad \text { and } \quad-\Delta \mathbf{y}^{\varepsilon}=\nabla p^{\varepsilon}+R a \hat{k} \theta^{\varepsilon}(0)
$$

and we have made the further approximation that $\theta^{\varepsilon}(t) \approx \theta^{\varepsilon}(0)$ relative to the slow time scale $\varsigma$.

Next, we return to the original time scale $t$ and obtain the effective dynamics for (2.1)-(2.2) starting from any initial condition $\left(\theta_{0}^{\varepsilon}, \mathbf{u}_{0}^{\varepsilon}\right) \in \mathbf{H}$,

$$
\begin{gathered}
-\Delta \tilde{\mathbf{u}}^{\varepsilon}=\nabla p^{\varepsilon}+R a \cdot \hat{\mathbf{k}} \tilde{\theta}^{\varepsilon}+\Delta \mathbf{w}^{\varepsilon}(t), \quad \nabla \cdot \tilde{\mathbf{u}}^{\varepsilon}=0, \\
d \tilde{\theta}^{\varepsilon}+\left(\tilde{\mathbf{u}}^{\varepsilon} \cdot \nabla \tilde{\theta}^{\varepsilon}-\Delta \tilde{\theta}^{\varepsilon}\right) d t=\tilde{R} a \cdot \tilde{u}_{d}^{\varepsilon} d t+\sum_{k=1}^{N} \sigma_{k} d W^{k}, \quad \tilde{\theta}^{\varepsilon}(0)=\theta_{0}^{\varepsilon},
\end{gathered}
$$

where $\mathbf{w}^{\varepsilon}$ solves

$$
\begin{aligned}
& \partial_{t} \mathbf{w}^{\varepsilon}-\frac{1}{\varepsilon} \Delta \mathbf{w}^{\varepsilon}=\frac{1}{\varepsilon} \nabla q^{\varepsilon}, \quad \nabla \cdot \mathbf{w}^{\varepsilon}=0, \\
& \mathbf{w}^{\varepsilon}(0)=\mathcal{P}_{N^{\varepsilon}} \mathbf{u}_{0}^{\varepsilon}+\mathbf{y}^{\varepsilon}, \quad \text { and } \quad-\Delta \mathbf{y}^{\varepsilon}=\nabla p^{\varepsilon}+R a \cdot \hat{\mathbf{k}} \theta_{0}^{\varepsilon}, \quad \nabla \cdot \mathbf{y}^{\varepsilon}=0 .
\end{aligned}
$$

We supplement (5.24)-(5.26) with boundary conditions (2.3). Note that for technical reasons we slightly modify the initial condition for $\mathbf{w}^{\varepsilon}$ compared to $(5.23)$ by taking $\tilde{\mathbf{u}}^{\varepsilon}(0)=\mathcal{P}_{N^{\varepsilon}} \mathbf{u}_{0}^{\varepsilon}$, where we recall that $\mathcal{P}_{N^{\varepsilon}}$ is the projection onto the first $N^{\varepsilon}$ modes of the Stokes operator $A$ as in (5.27) and $N^{\varepsilon}$ satisfies

$$
\varepsilon\left(\lambda_{N^{\varepsilon}}^{*}\right)^{2} \sim 1
$$

This specification $\tilde{\mathbf{u}}^{\varepsilon}(0)$ is only used to avoid regularity issues at the initial time in (5.24) and as such, a number of other modifications can be employed.

\subsubsection{Rigorous Error Estimates for the Corrector}

The following theorem asserts that (5.24)-(5.26) approximates (2.1)-(2.2) in the desired norms. 
Theorem 5.1. Fix any $\varepsilon>0$ choose $N_{\varepsilon}$ satisfying (5.27). Suppose we are given a sequence $\left\{\mu^{0, \varepsilon}\right\}_{\varepsilon \in(0,1)} \subset$ $\operatorname{Pr}(\mathbf{H})$ such that

$$
\sup _{0<\varepsilon \leq 1} \int_{\mathbf{H}}\left(\|\nabla \mathbf{u}\|^{2}+\exp \left(\eta\|\mathbf{u}\|^{2}+\|\theta\|_{L^{3}}^{2}\right)\right) d \mu^{0, \varepsilon}(\mathbf{u}, \theta)<\infty .
$$

For each $\varepsilon>0$ we consider a martingale solutions $\left(\mathbf{u}^{\varepsilon}, \theta^{\varepsilon}\right)$ of $(2.1)-(2.3)$ as in Proposition 2.1, (i). We suppose that each $\left(\mathbf{u}^{\varepsilon}, \theta^{\varepsilon}\right)$ has initial conditions distributed according to the distribution $\mu^{0, \varepsilon}$ and satisfies the uniform moment bound (2.12). In particular, for each $\varepsilon>0$ the corresponding martingale solution fixes a stochastic basis $\mathcal{S}$ and defines $\left(\theta_{0}^{\varepsilon}, \mathbf{u}_{0}^{\varepsilon}\right):=\left(\theta^{\varepsilon}(0), \mathbf{u}^{\varepsilon}(0)\right)$. Then,

(i) up to the specification of the stochastic basis $\mathcal{S}$ and the initial conditions $\left(\mathbf{u}_{0}^{\varepsilon}, \theta_{0}^{\varepsilon}\right)$, there exists a unique, adapted

$$
\tilde{\theta}^{\varepsilon} \in L^{2}\left(\Omega ; L_{l o c}^{2}([0, \infty) ; V) \cap C([0, \infty) ; H)\right)
$$

solving (5.24)-(5.26).

(ii) For any $t>0$ there is $a \gamma_{0}=\gamma_{0}(\tilde{R} a, R a, t)$ such that, for any $0<\gamma \leq \gamma_{0}, p \geq \gamma$, and $\varepsilon>0$,

$$
\mathbb{E}\left(\sup _{s \in[0, t]}\left\|\tilde{\theta}^{\varepsilon}(s)-\theta^{\varepsilon}(s)\right\|^{p}\right) \leq C \varepsilon^{\gamma}, \quad \mathbb{E}\left(\sup _{s \in[0, t]}\left\|\tilde{\mathbf{u}}^{\varepsilon}(s)-\mathbf{u}^{\varepsilon}(s)\right\|^{p}\right) \leq C \varepsilon^{\gamma / 4},
$$

where the constants $C=C(\tilde{R} a, R a, t, p)$ and $\gamma_{0}$ and are both independent of $\varepsilon>0$.

Proof. As in Proposition 2.1, (iii), (iv) the well posedness of (5.24)-(5.26) is standard and can be established along similar lines as one would for the 2D Stochastic Navier-Stokes equations. To see this observe that, although we are working in 3D, we have one more degree of smoothing in the constitutive law, (5.24), producing $\tilde{\mathbf{u}}^{\varepsilon}$ from $\tilde{\theta}^{\varepsilon}$ compared to Biot-Savart in the Navier-Stokes equation. We omit further details here again referring the reader to e.g. $[9,11,17]$.

To prove (5.29) we reuse many of the estimates from the proof of Theorem 2.1. Taking $\tilde{\mathbf{v}}^{\varepsilon}=\mathbf{u}^{\varepsilon}-\tilde{\mathbf{u}}^{\varepsilon}$ and $\tilde{\phi}^{\varepsilon}=\theta^{\varepsilon}-\tilde{\theta}^{\varepsilon}$, we have

$$
\partial_{t} \tilde{\phi}^{\varepsilon}-\Delta \tilde{\phi}^{\varepsilon}=\tilde{R} a \cdot \tilde{v}_{d}^{\varepsilon}-\tilde{\mathbf{v}}^{\varepsilon} \cdot \nabla \tilde{\theta}^{\varepsilon}-\mathbf{u}^{\varepsilon} \cdot \nabla \tilde{\phi}^{\varepsilon}, \quad \tilde{\phi}^{\varepsilon}(0)=0,
$$

and hence repeating the arguments leading to (5.5) we obtain the estimate

$$
\sup _{s \in[0, t]}\left\|\tilde{\phi}^{\varepsilon}(s \wedge \tau)\right\|^{2} \leq \sup _{s \in[0, t \wedge \tau]}\left(\left\|\tilde{\theta}^{\varepsilon}(s)\right\|_{L^{3}}^{2}+\tilde{R} a^{2}\right) \int_{0}^{t \wedge \tau}\left\|\nabla \tilde{\mathbf{v}}^{\varepsilon}\left(t^{\prime}\right)\right\|^{2} d t^{\prime}
$$

for any $t>0$ and any stopping time $\tau \geq 0$. By (5.24) and (2.1), $\tilde{\mathbf{v}}^{\varepsilon}$ satisfies

$$
\varepsilon\left(\partial_{t} \mathbf{u}^{\varepsilon}+\mathbf{u}^{\varepsilon} \cdot \nabla \mathbf{u}^{\varepsilon}\right)-\Delta \tilde{\mathbf{v}}^{\varepsilon}=\nabla q^{\varepsilon}+R a \hat{\mathbf{k}} \tilde{\phi}^{\varepsilon}-\Delta \mathbf{w}^{\varepsilon},
$$

where $\mathbf{w}^{\varepsilon}$ solves (5.26). Referring to (5.24) we have

$$
\tilde{\mathbf{u}}^{\varepsilon}=A^{-1}\left(\operatorname{RaP}\left(\hat{\mathbf{k}} \tilde{\theta}^{\varepsilon}\right)\right)-\mathbf{w}^{\varepsilon},
$$

and consequently (5.25) and (5.26) yield

$$
\begin{aligned}
d \tilde{\mathbf{u}}^{\varepsilon} & =-\partial_{t} \mathbf{w}^{\varepsilon}+R a A^{-1} P\left(\hat{\mathbf{k}} d \tilde{\theta}^{\varepsilon}\right) \\
& =-\frac{1}{\varepsilon}\left(\Delta \mathbf{w}^{\varepsilon}+\nabla q^{\varepsilon}\right) d t-R a A^{-1} P\left(\hat{\mathbf{k}}\left(\tilde{\mathbf{u}}^{\varepsilon} \cdot \nabla \tilde{\theta}^{\varepsilon}-\Delta \tilde{\theta}^{\varepsilon}-\tilde{R} a \cdot \tilde{u}_{d}^{\varepsilon}\right)\right) d t+R a \sum_{k=1}^{N} A^{-1} P\left(\hat{\mathbf{k}} \sigma_{k}\right) d W^{k} .
\end{aligned}
$$

Multiplying (5.31) by $\varepsilon^{-1}$, subtracting (5.33) and rearranging we obtain

$$
\begin{aligned}
d \tilde{\mathbf{v}}^{\varepsilon}-\frac{1}{\varepsilon} \Delta \tilde{\mathbf{v}}^{\varepsilon} d t= & \frac{1}{\varepsilon}\left(\nabla \tilde{q}^{\varepsilon}+R a \hat{\mathbf{k}} \tilde{\phi}^{\varepsilon}\right) d t+\left(R a A^{-1} P\left(\hat{\mathbf{k}}\left(\tilde{\mathbf{u}}^{\varepsilon} \cdot \nabla \tilde{\theta}^{\varepsilon}-\Delta \tilde{\theta}^{\varepsilon}-\tilde{R} a \cdot \tilde{u}_{d}^{\varepsilon}\right)\right)-\mathbf{u}^{\varepsilon} \cdot \nabla \mathbf{u}^{\varepsilon}\right) d t \\
& -R a \sum_{k=1}^{N} A^{-1} P\left(\hat{\mathbf{k}} \sigma_{k}\right) d W^{k}, \quad \tilde{\mathbf{v}}^{\varepsilon}(0)=\left(I-\mathcal{P}_{N}\right) \mathbf{u}_{0}^{\varepsilon},
\end{aligned}
$$


with $\nabla \cdot \tilde{\mathbf{v}}^{\varepsilon}=0$. Here note the close similarity between (5.8) and (5.34); in view of (5.32), (5.26) the primary distinction here is in the initial condition.

As above in (5.9), the Itō formula implies

$$
\begin{aligned}
d\left\|\tilde{\mathbf{v}}^{\varepsilon}\right\|^{2}+\frac{2}{\varepsilon}\left\|\nabla \tilde{\mathbf{v}}^{\varepsilon}\right\|^{2} d t= & \frac{2}{\varepsilon} R a\left\langle\tilde{\phi}^{\varepsilon}, \tilde{v}_{d}^{\varepsilon}\right\rangle d t+2\left\langle R a A^{-1} P\left(\hat{\mathbf{k}}\left(\tilde{\mathbf{u}}^{\varepsilon} \cdot \nabla \tilde{\theta}^{\varepsilon}-\Delta \tilde{\theta}^{\varepsilon}-\tilde{R} a \cdot \tilde{u}_{d}^{\varepsilon}\right)\right)-\mathbf{u}^{\varepsilon} \cdot \nabla \mathbf{u}^{\varepsilon}, \tilde{\mathbf{v}}^{\varepsilon}\right\rangle d t \\
& +R a^{2} \sum_{k=1}^{N}\left|A^{-1} P\left(\hat{\mathbf{k}} \sigma_{k}\right)\right|^{2} d t-2 R a \sum_{k=1}^{N}\left\langle A^{-1} P\left(\hat{\mathbf{k}} \sigma_{k}\right), \tilde{\mathbf{v}}^{\varepsilon}\right\rangle d W^{k} \\
:= & \left(T_{1}+T_{2}+T_{3}+T_{4}+T_{5}+T_{6}\right) d t+S d W .
\end{aligned}
$$

We now estimate (5.35) with bounds similar to (5.10)-(5.13). Here, bounds on $\mathbf{u}^{0}$ need to be replaced with appropriate estimates for $\tilde{\mathbf{u}}^{\varepsilon}$. For the terms $T_{2}, T_{4}$ we simply treat $\tilde{\mathbf{u}}^{\varepsilon}$ terms as

$$
\left\|\tilde{\mathbf{u}}^{\varepsilon}(t)\right\|^{2} \leq C\left(R a^{2}\left\|\tilde{\theta}^{\varepsilon}(t)\right\|^{2}+\|\mathbf{w}(t)\|^{2}\right) \leq C\left(R a^{2}\left\|\tilde{\theta}^{\varepsilon}(t)\right\|^{2}+\left\|\mathbf{u}_{0}^{\varepsilon}\right\|^{2}+R a^{2}\left\|\theta_{0}^{\varepsilon}\right\|^{2}\right) .
$$

The estimate (5.13) on the term $T_{5}$ involves an $L^{\infty}$ bound on $\tilde{\mathbf{u}}^{\varepsilon}$ and thus requires a bit more care. In this case

$$
\left\|\tilde{\mathbf{u}}^{\varepsilon}(t)\right\|_{L^{\infty}}^{2} \leq C\left\|\tilde{\mathbf{u}}^{\varepsilon}(t)\right\|_{H^{2}}^{2} \leq C\left(R a^{2}\left\|\tilde{\theta}^{\varepsilon}(t)\right\|^{2}+\|\mathbf{w}(t)\|_{H^{2}}^{2} .\right.
$$

By standard properties of analytic semigroups, the inverse Poincaré inequality, and the form of $\mathbf{w}(0)$ one has

$$
\begin{aligned}
\|\mathbf{w}(t)\|_{H^{2}}^{2} & =\left\|e^{A t / \varepsilon} \mathbf{w}(0)\right\|_{H^{2}}^{2} \leq C\|\mathbf{w}(0)\|_{H^{2}}^{2} \leq C R a^{2}\left\|\theta_{0}^{\varepsilon}\right\|^{2}+C\left\|\mathcal{P}_{N^{\varepsilon}} \tilde{\mathbf{u}}_{0}^{\varepsilon}\right\|_{H^{2}}^{2} \\
& \leq C R a^{2}\left\|\theta_{0}^{\varepsilon}\right\|^{2}+C\left(\lambda_{N^{\varepsilon}}^{*}\right)^{2}\left\|\tilde{\mathbf{u}}_{0}^{\varepsilon}\right\|^{2},
\end{aligned}
$$

where $C$ is independent of $\varepsilon \in(0,1)$. Combining these two estimate and again taking advantage of the cancelation from $\mathbf{u}^{\varepsilon}=\tilde{\mathbf{v}}^{\varepsilon}+\tilde{\mathbf{u}}^{\varepsilon}$

$$
\left|T_{5}\right| \leq \frac{1}{4 \varepsilon}\left\|\nabla \tilde{\mathbf{v}}^{\varepsilon}\right\|^{2}+4 \varepsilon\left\|\tilde{\mathbf{u}}^{\varepsilon}\right\|_{L^{\infty}}^{2}\left\|\mathbf{u}^{\varepsilon}\right\|^{2} \leq \frac{1}{4 \varepsilon}\left\|\nabla \tilde{\mathbf{v}}^{\varepsilon}\right\|^{2}+C\left(R a^{2}+1\right)\left(\left\|\theta_{0}^{\varepsilon}\right\|^{4}+\left\|\mathbf{u}_{0}^{\varepsilon}\right\|^{4}+\left\|\mathbf{u}^{\varepsilon}\right\|^{4}\right) .
$$

Observe that in comparison to (5.13), the additional power of $\varepsilon$ is used to cancel $\left(\lambda_{N^{\varepsilon}}^{*}\right)^{2}$.

Combining the analogues of (5.10)-(5.12) with (5.36) and using (5.36) with (5.35) we obtain

$$
\begin{aligned}
d\left\|\tilde{\mathbf{v}}^{\varepsilon}\right\|^{2}+\frac{1}{\varepsilon}\left\|\nabla \tilde{\mathbf{v}}^{\varepsilon}\right\|^{2} d t & \leq \frac{C R a^{2}}{\varepsilon}\left\|\tilde{\phi}^{\varepsilon}\right\|^{2} d t+C\left(1+R a^{4}\right)\left(1+\tilde{R} a^{2}\right)\left(\left\|\tilde{\theta}^{\varepsilon}\right\|^{4}+\left\|\theta_{0}^{\varepsilon}\right\|^{4}+\left\|\mathbf{u}_{0}^{\varepsilon}\right\|^{4}+\left\|\mathbf{u}^{\varepsilon}\right\|^{4}+1\right) d t \\
& -2 R a \sum_{k=1}^{N}\left\langle A^{-1}\left(P\left(\hat{\mathbf{k}} \sigma_{k}\right)\right), \tilde{\mathbf{v}}^{\varepsilon}\right\rangle d W^{k}
\end{aligned}
$$

where the constant $C>0$ is independent of $R a, \tilde{R} a$, and $\varepsilon>0$. We now use (5.39) with (5.30) and repeate the stopping time argument as in (5.17)-(5.20) to infer the first part of the (5.29). ${ }^{10}$

We turn next to the the convergence of the velocity fields, the second part of (5.29). We obtain from (5.35) and the pointwise bounds yielding the drift terms in (5.39) that

$$
\begin{aligned}
\left\|\tilde{\mathbf{v}}^{\varepsilon}(t)\right\|^{2} & \leq \exp \left(-\frac{t}{\varepsilon}\right)\left\|\tilde{\mathbf{v}}_{0}^{\varepsilon}\right\|^{2}+C \int_{0}^{t} \exp \left(-\frac{t-s}{\varepsilon}\right)\left(\frac{R a^{2}}{\varepsilon}\left\|\tilde{\phi}^{\varepsilon}(s)\right\|^{2}+\mathcal{R}_{\varepsilon}(s)\right) d s+\mathcal{X}_{\varepsilon}(t), \\
& \leq \exp \left(-\frac{t}{\varepsilon}\right)\left\|\tilde{\mathbf{v}}_{0}^{\varepsilon}\right\|^{2}+C R a^{2} \sup _{s \in[0, t]}\left\|\tilde{\phi}^{\varepsilon}(s)\right\|^{2}+\varepsilon \sup _{s \in[0, t]} \mathcal{R}_{\varepsilon}(s)+\mathcal{X}_{\varepsilon}(t),
\end{aligned}
$$

where

$$
\mathcal{R}_{\varepsilon}(t):=C\left(1+R a^{4}\right)\left(1+\tilde{R} a^{2}\right)\left(\left\|\tilde{\theta}^{\varepsilon}\right\|^{4}+\left\|\theta_{0}^{\varepsilon}\right\|^{4}+\left\|\mathbf{u}_{0}^{\varepsilon}\right\|^{4}+\left\|\mathbf{u}^{\varepsilon}\right\|^{4}+1\right)
$$

\footnotetext{
${ }^{10}$ Note that the loss of the $\varepsilon$ in front of the second term after the inequality in (5.39) in comparison (5.14) does not charge the ultimate outcome of this bound as we only required an $\varepsilon$-independent upper bound for $\mathcal{M}_{\varepsilon}$ in $(5.18)$.
} 
and

$$
\mathcal{X}_{\varepsilon}(t):=-2 R a \int_{0}^{t} \exp \left(-\frac{t-s}{\varepsilon}\right) \sum_{k=1}^{N}\left\langle A^{-1}\left(P\left(\hat{\mathbf{k}} \sigma_{k}\right)\right), \tilde{\mathbf{v}}^{\varepsilon}\right\rangle d W^{k}=: \int_{0}^{t} \exp \left(-\frac{t-s}{\varepsilon}\right) g(s) d W .
$$

Using the inverse Poincaré inequality and (5.27) one has

$$
\exp \left(-\frac{t}{\varepsilon}\right)\left\|\tilde{\mathbf{v}}_{0}^{\varepsilon}\right\|^{2} \leq\left\|\left(I-P_{N^{\varepsilon}}\right) \mathbf{u}_{0}^{\varepsilon}\right\|^{2} \leq C\left(\lambda_{N^{\varepsilon}}^{*}\right)^{-1}\left\|\nabla \mathbf{u}_{0}^{\varepsilon}\right\|^{2} \leq C \varepsilon\left\|\nabla \mathbf{u}_{0}^{\varepsilon}\right\|^{2} .
$$

Therefore combining (5.40) with (5.41), using the bound already obtained for $\left\|\tilde{\phi}^{\varepsilon}(s)\right\|$ in (5.29) and the uniform bounds (2.12), (5.28)

$$
\mathbb{E} \sup _{s \in[0, t]}\left\|\tilde{\mathbf{v}}^{\varepsilon}(t)\right\|^{p} \leq C\left(\varepsilon^{p / 2}+\varepsilon^{\gamma}\right)+C \mathbb{E} \sup _{s \in[0, t]}\left|\mathcal{X}_{\varepsilon}(s)\right|^{p / 2}
$$

for any $p>0$, where $\gamma=\min \left\{p, \gamma_{0}\right\}$ is obtained from the bound $\left\|\tilde{\phi}^{\varepsilon}(s)\right\|$ and the constant $C=C(R a, \tilde{R} a, t, p)$ is independent of $\varepsilon>0$.

In order to estimate $\mathcal{X}_{\varepsilon}$ observe that this process satisfies

$$
d \mathcal{X}_{\varepsilon}+\frac{1}{\varepsilon} \mathcal{X}_{\varepsilon} d t=g d W, \quad \mathcal{X}_{\varepsilon}(0)=0
$$

and hence, by the Itō lemma,

$$
d \mathcal{X}_{\varepsilon}^{2}+\frac{2}{\varepsilon} \mathcal{X}_{\varepsilon}^{2} d t=g^{2} d t+2 g \mathcal{X}_{\varepsilon} d W
$$

Consequently,

$$
\mathbb{E} \sup _{s \in[0, t]}\left|\mathcal{X}_{\varepsilon}(s)\right|^{p / 2} \leq C \mathbb{E} \sup _{s \in[0, t]}\left|\int_{0}^{s} g \mathcal{X}_{\varepsilon} d W\right|^{p / 4}+C \mathbb{E}\left(\int_{0}^{t} g^{2} d s\right)^{p / 4} .
$$

With the Burkholder-David-Gundy inequality and Young's inequality we have

$$
\mathbb{E} \sup _{s \in[0, t]}\left|\int_{0}^{s} g \mathcal{X}_{\varepsilon} d W\right|^{p / 4} \leq C \mathbb{E}\left(\int_{0}^{t} g^{2} \mathcal{X}_{\varepsilon}^{2} d s\right)^{p / 8} \leq \frac{1}{2} \mathbb{E} \sup _{s \in[0, t]}\left|\mathcal{X}_{\varepsilon}\right|^{p / 2}+C \mathbb{E}\left(\int_{0}^{t}\left\|\tilde{\mathbf{v}}^{\varepsilon}\right\|^{2} d s\right)^{p / 4} .
$$

With (5.43), (5.44), the bound (5.45) now yields

$$
\mathbb{E} \sup _{s \in[0, t]}\left\|\tilde{\mathbf{v}}^{\varepsilon}(t)\right\|^{p} \leq C\left(\varepsilon^{p / 2}+\varepsilon^{\gamma}\right)+C \mathbb{E}\left(\int_{0}^{t}\left\|\tilde{\mathbf{v}}^{\varepsilon}\right\|^{2} d s\right)^{p / 4} .
$$

On the other hand, from (5.39), using for a second time the existing bounds on $\left\|\tilde{\phi}^{\varepsilon}(s)\right\|$ in (5.29) and (2.12), we have

$$
\mathbb{E} \int_{0}^{t}\left\|\nabla \tilde{\mathbf{v}}^{\varepsilon}\right\|^{2} d t \leq \varepsilon \mathbb{E}\left\|\tilde{\mathbf{v}}_{0}^{\varepsilon}\right\|^{2}+C R a^{2} \int_{0}^{t} \mathbb{E}\left\|\tilde{\phi}^{\varepsilon}\right\|^{2} d s+\varepsilon \mathbb{E} \int_{0}^{t} \mathcal{R}_{\varepsilon} d s \leq C\left(\varepsilon+\varepsilon^{\gamma}\right)
$$

where the constant $C$ depends on $t, R a, \tilde{R a}$ but again is independent of $\varepsilon>0$. When $p \leq 4$ the second portion of the desired inequality (5.29) now follows from (5.45), (5.46), and Hölder's inequality. On the other hand, when $p>4$ then we estimate

$$
\mathbb{E}\left(\int_{0}^{t}\left\|\tilde{\mathbf{v}}^{\varepsilon}\right\|^{2} d s\right)^{p / 4} \leq \mathbb{E} \sup _{s \in[0, t]}\left\|\tilde{\mathbf{v}}^{\varepsilon}\right\|^{p-2}+\mathbb{E} \int_{0}^{t}\left\|\tilde{\mathbf{v}}^{\varepsilon}\right\|^{2} d s \leq \sup _{s \in[0, t]}\left\|\tilde{\mathbf{v}}^{\varepsilon}\right\|^{p-2}+C\left(\varepsilon+\varepsilon^{\gamma}\right),
$$

so that the second part of (5.29) follows in this later case with (5.46) and an iterative argument. This completes the proof of Theorem 5.1, (ii). 


\section{Convergence of the Nusselt number}

In this final section we prove Corollary 1.1, note that this is not an immediate consequence of Theorem 2.2 . Indeed, the Nusselt number $N u$ (cf. (1.8)) is defined as a statistical average of the observable

$$
\phi_{N u}(\mathbf{u}, \theta)=\frac{1}{|\mathcal{D}|} \int_{\mathcal{D}} u_{2} \theta d x
$$

but (2.30) is not satisfied since $\phi_{N u} \notin V(\mathbf{H})$. Nevertheless, Corollary 1.1 follows from a combination of Theorem 2.2 with exponential moment bounds on the unique invariant measures.

Proof of Corollary 1.1. In two space dimensions, with $N=\infty$ and $\operatorname{Pr}=\operatorname{Pr}(\operatorname{Ra}, \tilde{R} a)$ large enough (or $\varepsilon=\operatorname{Pr}^{-1}$ small enough), [22, Theorem 1.3] and Theorem 2.2 yields respectively unique invariant measures $\mu_{\varepsilon}$ of $(2.1)-(2.2)$ and $\mu_{0}$ for $(2.4)-(2.5)$. We aim to show that $\lim _{\varepsilon \rightarrow 0}(N u)_{\varepsilon}=(N u)_{0}$. To simplify the notation, write $\phi$ instead of $\phi_{N u}$ defined by (6.1).

Consider a smooth cut-off function $\psi:[0, \infty) \rightarrow[0,1]$ satisfying $\psi \equiv 1$ for $x \in[0,1], \psi \equiv 0$ for $x \geq 2$, and $\left|\psi^{\prime}\right| \leq 2$ everywhere. Denote $\psi_{R}(\cdot):=\psi(\cdot / R)$ and write

$$
\begin{aligned}
& \left|\int_{\mathbf{H}} \phi(\mathbf{u}, \theta) d \mu_{\varepsilon}(\mathbf{u}, \theta)-\int_{H} \phi(L(\theta)) d \mu_{0}(\theta)\right| \\
& \leq\left|\int_{\mathbf{H}} \phi(\mathbf{u}, \theta) \psi_{R}\left(\|\mathbf{u}\|^{2}+\|\theta\|^{2}\right) d \mu_{\varepsilon}(\mathbf{u}, \theta)-\int_{H} \phi(L(\theta)) \psi_{R}\left(\|L(\theta)\|^{2}\right) d \mu_{0}(\theta)\right| \\
& \quad+\left|\int_{\mathbf{H}} \phi(\mathbf{u}, \theta)\left(1-\psi_{R}\left(\|\mathbf{u}\|^{2}+\|\theta\|^{2}\right)\right) d \mu_{\varepsilon}(\mathbf{u}, \theta)\right|+\left|\int_{H} \phi(L(\theta))\left(1-\psi_{R}\left(\|L(\theta)\|^{2}\right)\right) d \mu_{0}(\theta)\right| \\
& =: I_{1}+I_{2}+I_{3} .
\end{aligned}
$$

We bound the first term by observing that $\phi_{R}(\mathbf{u}, \theta):=\phi(\mathbf{u}, \theta) \psi_{R}\left(\|\mathbf{u}\|^{2}+\|\theta\|^{2}\right)$ satisfies

$$
\left[\phi_{R}\right]_{\eta} \leq C(R+1)
$$

for a constant $C>0$. It follows from (6.2) and Theorem 2.2 that $I_{1} \leq \tilde{C}(R+1) \varepsilon^{\tilde{q}}$. We control $I_{2}$ using Markov's inequality and (2.13), which yields, for any $\eta>0$ sufficiently small

$$
\mu_{\varepsilon}\left(E_{R}^{c}\right) \leq e^{-\eta R} \int_{\mathbf{H}} \exp \left(\eta\left(\|\mathbf{u}\|^{2}+\|\theta\|^{2}\right)\right) d \mu_{\varepsilon}(\mathbf{u}, \theta) \leq C_{0} e^{-\eta R} .
$$

uniformly in $\varepsilon>0$, where $E_{R}=\left\{\|\mathbf{u}\|^{2}+\|\theta\|^{2} \leq R\right\}$. Notice that $1-\psi_{R}\left(\|u\|^{2}+\|\theta\|^{2}\right) \leq \mathbb{1}_{E_{R}^{c}}$, and observe that for fixed $\eta>0$, on the set $E_{R}^{c}$ we have $\left|\phi_{N u}(\mathbf{u}, \theta)\right| \leq \exp \left(\eta\left(\|\mathbf{u}\|^{2}+\|\theta\|^{2}\right) / 2\right)$ for $R=R(\eta)$ sufficiently large. By Hölder's inequality we obtain

$$
I_{2} \leq C\left(\int_{\mathbf{H}} \exp \left(\eta\left(\|\mathbf{u}\|^{2}+\|\theta\|^{2}\right)\right) d \mu_{\varepsilon}(\mathbf{u}, \theta)\right)^{1 / 2}\left(\mu_{\varepsilon}\left(E_{R}^{c}\right)\right)^{1 / 2} \leq C C_{0}^{1 / 2} e^{-\eta R / 2} .
$$

We can control $I_{3}$ similarly by using exponential moment bounds for the invariant measure $\mu_{0}$, and the estimate $\|L(\theta)\| \leq C\|\theta\|$. Thus,

$$
\left|\int_{\mathbf{H}} \phi_{N u(\varepsilon)}(\mathbf{u}, \theta) d \mu_{\varepsilon}(\mathbf{u}, \theta)-\int_{H} \phi_{N u(0)}(L(\theta)) d \mu_{0}(\theta)\right| \leq C\left((R+1) \varepsilon^{\tilde{q}}+e^{-\eta R / 2}\right),
$$

and we can make this expression less than any $\delta>0$ by taking $R=R(\delta)$ sufficiently large and then $\varepsilon=\varepsilon(R, \delta)$ sufficiently small.

Remark 6.1. From the proof above we can easily infer that Corollary 1.1 will also apply to any observable $\phi$ on the extended phase space that is locally Lipschitz and sub-exponential at infinity (for a sufficiently small exponential power $\eta>0$ dictated by (2.13)). We have chosen the convergence of the Nusselt number to emphasize the physical significance of our results. 


\section{A Appendix: Moment Bounds for Stochastic Drift-Diffusion Equa- tions}

In this appendix we collect some moment bounds proved in [22] which have been used in the analysis above.

As in [22] we consider the following class of stochastic divergence-free drift diffusion systems

$$
d \xi+\mathbf{v} \cdot \nabla \xi d t=\left(\tilde{R} a \cdot v_{3}+\Delta \xi\right) d t+\sum_{k=1}^{N} \sigma_{k} d W^{k}, \quad \xi(0)=\xi_{0}
$$

evolving on the three dimensional domain $\mathcal{D}=[0, L]^{2} \times[0,1]$. Here $\tilde{R} a>0$ is a fixed parameter and $\mathbf{v}=\left(v_{1}, v_{2}, v_{3}\right)$ is any sufficiently regular and adapted, divergence free vector field. Both $\mathbf{v}$ and $\xi$ are supposed to satisfy the boundary condition (2.3). Recall that by the change of variable $T=\xi+\tilde{R} a(1-z)$ we may reformulate (A.1) as

$$
d T+\mathbf{v} \cdot \nabla T d t=\Delta T d t+\sum_{k=1}^{N} \sigma_{k} d W^{k}, \quad T(0)=T_{0}=\xi_{0}+\tilde{R} a(1-z),
$$

where $\mathbf{v}$ and $T$ satisfy boundary conditions (1.3). As such, bounds for $\xi$ solving (A.1) immediately translate to bounds for $T$.

In [22] we prove:

Proposition A.1. Suppose that $\mathbf{v} \in L_{\text {loc }}^{2}\left([0, \infty) ; V_{1} \cap\left(H^{2}(\mathcal{D})\right)^{3}\right) \cap C\left([0, \infty) ; H_{1}\right)$ a.s. and is $\mathcal{F}_{t}$-adapted. Fix any $p \geq 2$ and any initial condition $\xi_{0} \in H \cap L^{p}(\mathcal{D})$ which is $\mathcal{F}_{0}$ measurable with

$$
\mathbb{E} \exp \left(\eta\left\|\xi_{0}\right\|_{L^{p}}^{2}\right)<\infty,
$$

for some $\eta>0$. Then there exists $\eta_{0}=\eta_{0}(\sigma, \tilde{R} a, p)>0$ such that for any $t \geq 0$ and any positive $\eta \leq \eta_{0}$,

$$
\mathbb{E} \exp \left(\frac{\eta}{2^{p / 2+2}} \sup _{s \in[0, t]}\|\xi\|_{L^{p}}^{2}\right) \leq C_{1} \mathbb{E} \exp \left(\eta\left\|\xi_{0}\right\|_{L^{p}}^{2}+\eta p t\left(\|\sigma\|_{L^{p}}^{2}+2^{p / 2}\left(4 \tilde{R} a^{2}+1\right)\right)\right)
$$

for a constant $C=C(\tilde{R} a, p)$ independent of $t, \eta, \xi_{0}$, and $\mathbf{v}$. Furthermore,

$$
\mathbb{E} \exp \left(\frac{\eta}{2^{p / 2+2}}\|\xi(t)\|_{L^{p}}^{2}\right) \leq C \mathbb{E} \exp \left(\eta\left(e^{-\kappa t}\left\|\xi_{0}\right\|^{2}\right)\right)
$$

where again $C=C\left(\tilde{R} a, p,\|\sigma\|_{L^{p}}, \mathcal{D}\right)$ and $\kappa=\kappa(\tilde{R} a, \mathcal{D})>0$ are independent of $t, \eta, \xi_{0}$, and $\mathbf{v}$.

We now return to the infinite Prandtl system (2.4)-(2.5) and recall a bound analogous to (A.4) but which uses more of the specific structure of the velocity equation.

Proposition A.2. Fix an initial condition $\theta_{0}^{0} \in H$ which is $\mathcal{F}_{0}$ measurable, and let $\theta^{0}=\theta^{0}\left(t, \theta_{0}^{0}\right)$ denote the corresponding solution to (2.4)-(2.5). There is a universal constant $\eta^{*}>0$ such that for any $t>0$ and $\eta \in\left(0, \eta^{*}\right]$, there exists $C=C(R a, \tilde{R} a)>0$ such that

$$
\mathbb{E}\left(\exp \left(\eta\left\|\theta^{0}\right\|^{2}+\frac{\eta e^{-t / 4}}{4} \int_{0}^{t}\left\|\nabla \theta^{0}\right\|^{2} d s\right)\right) \leq C \exp \left(\eta(1+4 R a \tilde{R} a) e^{-t / 2}\left\|\theta_{0}^{0}\right\|^{2}\right) .
$$

The proof of Proposition A.2 can be found in [22].

\section{B Gradient Estimates on the Markov Semigroup}

In this section we establish the gradient bound for the Markov semigroup generated by (2.4)-(2.5) in order to prove (4.2). For this purpose we begin by briefly recalling how (4.2) is translated to a control problem 
through the use of Malliavin calculus. We refer to e.g. [36] or [35] for further general background on this subject and to $[21,26,28]$ for the application of this formalism in a setting close to ours.

Define the random operators

$$
\mathcal{J}_{0, t} \xi:=\lim _{\epsilon \rightarrow 0} \frac{\theta^{0}\left(t, \theta_{0}+\epsilon \xi, W\right)-\theta^{0}\left(t, \theta_{0}, W\right)}{\epsilon}
$$

for any $\xi \in H$ and

$$
\mathcal{A}_{0, t} w:=\lim _{\epsilon \rightarrow 0} \frac{\theta^{0}\left(t, \theta_{0}, W+\epsilon \int_{0}^{\cdot} w\right)-\theta^{0}\left(t, \theta_{0}, W\right)}{\epsilon}
$$

for any $w \in L^{2}\left(\Omega ; L^{2}\left([0, t] ; \mathbb{R}^{N}\right)\right)$. Here $\mathcal{A}_{0, t} w=\left\langle\mathfrak{D} \theta^{0}, w\right\rangle$, where the unbounded operator $\mathfrak{D}: L^{2}(\Omega ; H) \mapsto$ $L^{2}\left(\Omega ; L^{2}\left(0, t, \mathbb{R}^{N}\right) \otimes H\right)$ is the Malliavin derivative and $w$ is any element in the domain of the dual operator $\delta$ of $\mathfrak{D}$.

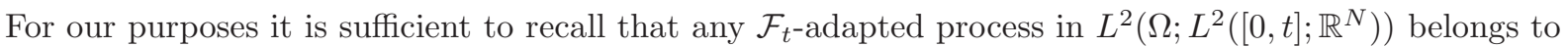
the domain of $\delta$ and $\delta(w)$ corresponds to the Itō integral of $w$ so that

$$
\mathbb{E}\langle\mathfrak{D} X, w\rangle=\mathbb{E}\left(X \int_{0}^{t} w d W\right)
$$

for any $X \in \operatorname{Dom}(\mathfrak{D})$ and any $\mathcal{F}_{t}$-adapted $w$. This is a special case of the Malliavin integration by parts formula. We furthermore recall that $\mathfrak{D}$ satisfies a chain rule namely that if $\phi \in C^{1}(H)$ and $\theta \in \operatorname{Dom}(\mathfrak{D})$ then $\phi(\theta) \in \operatorname{Dom}(\mathfrak{D})$ and

$$
\mathfrak{D} \phi(\theta)=\nabla \phi(\theta) \mathfrak{D} \theta .
$$

Combining (B.3)-(B.4) and making use of the Itō isometry we infer that,

$$
\begin{aligned}
\nabla P_{t}^{0} \phi\left(\theta_{0}\right) \xi & =\mathbb{E}\left(\nabla \phi\left(\theta^{0}\left(t, \theta_{0}\right)\right) \mathcal{J}_{0, t} \xi\right)=\mathbb{E}\left(\phi\left(\theta^{0}\left(t, \theta_{0}\right)\right) \int_{0}^{t} w d W\right)+\mathbb{E}\left(\nabla \phi\left(\theta^{0}\left(t, \theta_{0}\right)\right)\left(\mathcal{J}_{0, t} \xi-\mathcal{A}_{0, t} w\right)\right) \\
& \leq \sqrt{P_{t}^{0}\left(|\phi(\theta)|^{2}\right)}\left(\mathbb{E} \int_{0}^{t}|w|^{2} d t\right)^{1 / 2}+\sqrt{P_{t}^{0}\left(\|\nabla \phi(\theta)\|^{2}\right)}\left(\mathbb{E}\left\|\mathcal{J}_{0, t} \xi-\mathcal{A}_{0, t} w\right\|^{2}\right)^{1 / 2}
\end{aligned}
$$

for any $\phi \in C_{b}^{1}(H), \theta_{0} \in H$ and any (adapted) $w \in L^{2}\left(\Omega ; L^{2}\left([0, t] ; \mathbb{R}^{N}\right)\right)$.

Our desired bound (4.2) follows from (B.5) if, for every $\xi \in H$ with $\|\xi\|=1$ there is (adapted) $w=$ $w(\xi) \in L^{2}\left([0, \infty) ; \mathbb{R}^{N}\right)$ such that

$$
\begin{aligned}
& \mathbb{E}\left\|\mathcal{J}_{0, t} \xi-\mathcal{A}_{0, t} w(\xi)\right\|^{2} \leq C \exp \left(2 \eta\left\|\theta_{0}\right\|^{2}\right) \delta(t), \\
& \sup _{\|\xi\|=1} \mathbb{E} \int_{0}^{\infty}|w(\xi)|^{2} d t \leq C \exp \left(2 \eta\left\|\theta_{0}\right\|^{2}\right)
\end{aligned}
$$

where $\delta(t) \rightarrow 0$ as $t \rightarrow \infty$ and $C, \eta$, and $\delta$ are independent of $\theta_{0}$.

To solve the control problem (B.6)-(B.7) we observe that (B.1) and (B.2) admit explicit characterizations as linearizations of $(2.4)-(2.5)$. For any $\xi \in H^{0}$ we let $\rho(t)=\rho(t, \xi):=\mathcal{J}_{0, t} \xi$, which satisfies

$$
\partial_{t} \rho+\mathbf{u}^{0} \cdot \nabla \rho+\mathbf{v}^{0} \cdot \nabla \theta^{0}=\tilde{R} a \cdot v_{d}^{0}+\Delta \rho, \quad-\Delta \mathbf{v}^{0}=\nabla p+\operatorname{Ra} \hat{\mathbf{k}} \rho, \quad \nabla \cdot \mathbf{v}^{0}=0, \quad \rho(0)=\xi,
$$

supplemented by boundary conditions as in (2.3). ${ }^{11}$ On the other hand, setting $\tilde{\rho}:=\mathcal{A}_{0, t} w$ for any $w \in$ $L^{2}\left([0, t], \mathbb{R}^{N}\right)$ we have

$$
\partial_{t} \tilde{\rho}+\mathbf{u}^{0} \cdot \nabla \tilde{\rho}+\tilde{\mathbf{v}}^{0} \cdot \nabla \theta^{0}=\tilde{R} a \cdot v_{d}^{0}+\Delta \tilde{\rho}+\sum_{k=1}^{N} \sigma_{k} w_{k},-\Delta \tilde{\mathbf{v}}^{0}=\nabla p+\operatorname{Ra} \hat{\mathbf{k}} \tilde{\rho}, \nabla \cdot \tilde{\mathbf{v}}^{0}=0, \tilde{\rho}(0)=0,
$$

\footnotetext{
11 Notice that (B.8) can also be written as

$$
\partial_{t} \rho+\left(L \theta^{0}\right) \cdot \nabla \rho+(L \rho) \cdot \nabla \theta^{0}=\tilde{R a}(L \rho)+\Delta \rho, \quad \rho(0)=\xi,
$$
}

where $L=R a A^{-1} P \hat{k}$ and $A$ is the Stokes operator, $P$; cf. (5.1) and (2.6) above. Similar formulations can also be given for (B.9), (B.10). 
again with boundary conditions as in (2.3).

Denote $\bar{\rho}(t)=\bar{\rho}(t, \xi, w)=\rho-\tilde{\rho}$ and $\overline{\mathbf{v}}:=\mathbf{v}-\tilde{\mathbf{v}}$ for any $w \in L^{2}\left([0, \infty) ; \mathbb{R}^{N}\right)$ and $\xi \in H$. We now choose $w$ as a function of $\xi$ as follows. Let $P_{N}$ be the projection on the first $N$ eigenfunctions of the Laplacian with boundary conditions as in (2.3). Set $w(t):=\sigma^{-1} \lambda P_{N} \bar{\rho}$, where $\lambda>0$ and $N$ will be selected below. ${ }^{12}$ Relative to this choice of $w=w(\xi), \bar{\rho}$ satisfies

$$
\partial_{t} \bar{\rho}+\mathbf{u}^{0} \cdot \nabla \bar{\rho}+\overline{\mathbf{v}}^{0} \cdot \nabla \theta^{0}=\tilde{R} a \cdot \bar{v}_{d}^{0}+\Delta \bar{\rho}-\lambda P_{N} \bar{\rho}, \quad \Delta \overline{\mathbf{v}}^{0}=\nabla p+\operatorname{Ra} \hat{\mathbf{k}} \bar{\rho}, \quad \nabla \cdot \overline{\mathbf{v}}^{0}=0, \quad \bar{\rho}(0)=\xi .
$$

Testing (B.10) with $\bar{\rho}$ and $\overline{\mathbf{v}}^{0}$ respectively, and using that both $\mathbf{u}^{0}$ and $\overline{\mathbf{v}}^{0}$ are divergence free vector fields, we obtain

$$
\frac{d}{d t}\|\bar{\rho}\|^{2}+2\|\nabla \bar{\rho}\|^{2}+2 \lambda\left\|P_{N} \bar{\rho}\right\|^{2}=2 \int_{\mathcal{D}}\left(\tilde{R} a \bar{v}_{d}^{0}-\overline{\mathbf{v}}^{0} \cdot \nabla \theta^{0}\right) \bar{\rho} d x
$$

and

$$
\left\|\nabla \overline{\mathbf{v}}^{0}\right\| \leq \mathrm{Ra}\|\bar{\rho}\| .
$$

With standard Sobolev embeddings and (B.12) we have, for any $\eta>0$,

$$
\begin{aligned}
\left|\int_{\mathcal{D}}\left(\tilde{R} a \bar{v}_{d}^{0}-\overline{\mathbf{v}}^{0} \cdot \nabla \theta^{0}\right) \bar{\rho} d x\right| & \leq\left\|\overline{\mathbf{v}}^{0}\right\|_{L^{6}}\left\|\nabla \theta^{0}\right\|\|\bar{\rho}\|_{L^{3}}+\tilde{R} a\left\|\overline{\mathbf{v}}^{0}\right\|\|\bar{\rho}\| \\
& \leq \tilde{C}\left\|\nabla \overline{\mathbf{v}}^{0}\right\|\left\|\nabla \theta^{0}\right\|\|\bar{\rho}\|^{1 / 2}\|\nabla \bar{\rho}\|^{1 / 2}+\tilde{R} a\left\|\nabla \overline{\mathbf{v}}^{0}\right\|\|\bar{\rho}\| \\
& \leq \tilde{C} R a\left\|\nabla \theta^{0}\right\|\|\bar{\rho}\|^{3 / 2}\|\nabla \bar{\rho}\|^{1 / 2}+\tilde{C} R a \tilde{R} a\|\bar{\rho}\|^{2} \\
& \leq\|\nabla \bar{\rho}\|^{2}+\left(\tilde{C}(R a)^{4 / 3}\left\|\nabla \theta^{0}\right\|^{4 / 3}+\tilde{C} R a \tilde{R} a\right)\|\bar{\rho}\|^{2} \\
& \leq\|\nabla \bar{\rho}\|^{2}+\left(\eta\left\|\nabla \theta^{0}\right\|^{2}+C\right)\|\bar{\rho}\|^{2},
\end{aligned}
$$

where $C=C(\operatorname{Ra}, \tilde{R} a, \eta)=\frac{\tilde{C} R a^{4}}{\eta^{2}}+\tilde{C} R a \tilde{R} a$ and $\tilde{C}$ is a universal constant. Also since $P_{N}$ and $-\Delta$ commute we have for $Q_{N}:=I-P_{N}$

$$
\|\nabla \bar{\rho}\|^{2}=-\left\langle P_{N} \bar{\rho}, \Delta P_{N} \bar{\rho}\right\rangle-\left\langle Q_{N} \bar{\rho}, \Delta Q_{N} \bar{\rho}\right\rangle=\left\|\nabla P_{N} \bar{\rho}\right\|^{2}+\left\|\nabla Q_{N} \bar{\rho}\right\|^{2} \geq\left\|\nabla Q_{N} \bar{\rho}\right\|^{2} \geq \lambda_{N}\left\|Q_{N} \bar{\rho}\right\|^{2},
$$

where the last inequality follows from the generalized Poincaré inequality. Choose $2 \lambda=\lambda_{N}$ (with $N$ to be chosen below) and combine (B.11) and (B.13) to infer

$$
\frac{d}{d t}\|\bar{\rho}\|^{2}+\left(\lambda_{N}-\left(\eta_{0}\left\|\nabla \theta^{0}\right\|^{2}+C\right)\right)\|\bar{\rho}\|^{2} \leq 0,
$$

and hence, since $\bar{\rho}(0)=\xi$,

$$
\|\bar{\rho}(t)\|^{2} \leq\|\xi\|^{2} \exp \left(\eta_{0} \int_{0}^{t}\left\|\nabla \theta^{0}\right\|^{2} d r+\left(C-\lambda_{N}\right) t\right) .
$$

Applying Proposition A.1 we conclude that, for any $\theta_{0}^{0} \in H$, and $\eta \in\left(0, \eta_{0}\right]$,

$$
\mathbb{E}\|\bar{\rho}(t)\|^{2} \leq C\|\xi\|^{2} \exp \left(\eta\left\|\theta_{0}^{0}\right\|^{2}+\left(C+\eta-\lambda_{N}\right) t\right),
$$

where $C=C(\operatorname{Ra}, \tilde{R a})$ is independent of $\xi$ and $\theta_{0}^{0}$ and $t \geq 0$. By now choosing $N$ large enough such that $\lambda_{N}>2\left(C+\eta\|\sigma\|^{2}\right)$ we obtain

$$
\mathbb{E}\|\bar{\rho}(t)\|^{2} \leq C\|\xi\|^{2} \exp \left(\eta\left\|\theta_{0}^{0}\right\|^{2}-\frac{\lambda_{N}}{2} t\right)
$$

where $C=C(\operatorname{Ra}, \tilde{R} a)$ is independent of $\xi$ and $\theta_{0}^{0}$ and $t \geq 0$. This yields the first bound (B.6).

To obtain the second desired bound, (B.7), we use (B.16) to estimate

$$
\mathbb{E} \int_{0}^{\infty}|w(\xi)|^{2} d t=\left\|\sigma^{-1}\right\|^{2} \lambda_{N}^{2} \mathbb{E} \int_{0}^{\infty}\left\|P_{N} \bar{\rho}\right\|^{2} d t \leq C \exp \left(\eta\left\|\theta_{0}^{0}\right\|^{2}\right),
$$

where $C=C\left(\lambda_{N}, R a, \tilde{R} a\right)$ is independent of $\theta_{0}^{0}$ yielding (B.7). The bound (4.2) now follows.

\footnotetext{
${ }^{12}$ Of course the choice of $N$ will determine the number of modes subject to stochastic perturbation. Observe that $w$ is well defined as $\left\{\sigma_{k}\right\}_{k=1}^{N}$ is the set of the first $N$ (nonzero) eigenvectors of the Laplacian.
} 
Remark B.1. We can use the same argument leading to (B.15) to show that

$$
\|\rho(t)\|^{2} \leq\|\xi\|^{2} \exp \left(\eta \int_{0}^{t}\left\|\nabla \theta^{0}\right\|^{2} d r+C t\right)
$$

that is, for any $\eta>0$,

$$
\left\|\mathcal{J}_{0, t}\right\| \leq \exp \left(\eta \int_{0}^{t}\left\|\nabla \theta^{0}\right\|^{2} d r+C t\right)
$$

where, as above, $C=C(R a, \tilde{R} a)=\frac{\tilde{C} R a^{4}}{\eta^{2}}+R a \tilde{R} a$.

Remark B.2. Using Proposition A.2 and (B.17) we can easily establish the Lyapunov bound (4.1) with

$$
C_{1}=\exp \left(\frac{C R a^{4} e^{t^{*} / 2}}{\eta^{2}}+R a \tilde{R} a\right)
$$

\section{Acknowledgments}

The majority of this work was carried out during a Research in Peace (RIP) fellowship at the Institut Mittag-Leffler, Stockholm, Sweden. We are grateful to this institution for providing us with a unique working environment to continue our ongoing collaborations. JF and GR would like to acknowledge Virginia Tech for facilitating a research visit during which this work was finalized. We would also like to extend our warm thanks to P. Constantin, C. Doering, S. Friedlander, J. Mattingly, E. Thomann, and J. Whitehead for stimulating feedback on this work. NEGH was partially supported in this work under the grants NSF-DMS1313272, Simons-515990.

\section{References}

[1] G. Ahlers, S. Grossmann, and D. Lohse. Heat transfer and large scale dynamics in turbulent Rayleigh-Bénard convection. Reviews of Modern Physics, 81(2):503, 2009.

[2] A. Barletta and D. A. Nield. On the Rayleigh-Bénard-Poiseuille problem with internal heat generation. International Journal of Thermal Sciences, 57:1-16, 2012.

[3] E. Bodenschatz, W. Pesch, and G. Ahlers. Recent developments in Rayleigh-Bénard convection. In Annual review of fluid mechanics, Vol. 32, volume 32 of Annu. Rev. Fluid Mech., pages 709-778. Annual Reviews, Palo Alto, CA, 2000.

[4] M. Breuer and U. Hansen. Turbulent convection in the zero Reynolds number limit. Euro. phys. Lett., 86(24004), 2009.

[5] S. Cerrai and N. E. Glatt-Holtz. On the convergence of stationary solutions in the Smoluchowski-Kramers approximation of infinite dimensional systems. 2018. (to appear).

[6] P. Constantin and C. R. Doering. Infinite Prandtl number convection. J. Statist. Phys., 94(1-2):159-172, 1999.

[7] P. Constantin and C. Foias. Navier-Stokes equations. Chicago Lectures in Mathematics. University of Chicago Press, Chicago, IL, 1988.

[8] P. Constantin, N. Glatt-Holtz, and V. Vicol. Unique ergodicity for fractionally dissipated, stochastically forced 2d euler equations. Communications in Mathematical Physics, 330(2):819-857, 2014.

[9] G. Da Prato and J. Zabczyk. Stochastic equations in infinite dimensions, volume 44 of Encyclopedia of Mathematics and its Applications. Cambridge University Press, Cambridge, 1992.

[10] G. Da Prato and J. Zabczyk. Ergodicity for infinite-dimensional systems, volume 229 of London Mathematical Society Lecture Note Series. Cambridge University Press, Cambridge, 1996.

[11] A. Debussche, N. Glatt-Holtz, and R. Temam. Local martingale and pathwise solutions for an abstract fluids model. Physica D, 240(14-15):1123-1144, 2011.

[12] C. R. Doering and P. Constantin. On upper bounds for infinite Prandtl number convection with or without rotation. J. Math. Phys., 42(2):784-795, 2001.

[13] J. L. Doob. Asymptotic properties of Markoff transition prababilities. Trans. Amer. Math. Soc., 63:393-421, 1948. 
[14] R. M. Dudley. Real analysis and probability, volume 74. Cambridge University Press, 2002.

[15] W. E and J. C. Mattingly. Ergodicity for the Navier-Stokes equation with degenerate random forcing: finite-dimensional approximation. Comm. Pure Appl. Math., 54(11):1386-1402, 2001.

[16] W. E, J. C. Mattingly, and Y. G.. Sinai. Gibbsian dynamics and ergodicity for the stochastically forced Navier-Stokes equation. Comm. Math. Phys., 224(1):83-106, 2001. Dedicated to Joel L. Lebowitz.

[17] F. Flandoli and D. Gatarek. Martingale and stationary solutions for stochastic Navier-Stokes equations. Probab. Theory Related Fields, 102(3):367-391, 1995.

[18] C. Foiaş and G. Prodi. Sur le comportement global des solutions non-stationnaires des équations de Navier-Stokes en dimension 2. Rend. Sem. Mat. Univ. Padova, 39:1-34, 1967.

[19] C. Foias, O. Manley, R. Rosa, and R. Temam. Navier-Stokes equations and turbulence, volume 83 of Encyclopedia of Mathematics and its Applications. Cambridge University Press, Cambridge, 2001.

[20] J. Földes, S. Friedlander, N. Glatt-Holtz, and G. Richards. Asymptotic Analysis for Randomly Forced MHD. arXiv preprint arXiv:1604.06352, 2016. Available at http://arxiv.org/abs/1604.06352.

[21] J. Földes, N. Glatt-Holtz, G. Richards, and E. Thomann. Ergodic and mixing properties of the Boussinesq equations with a degenerate random forcing. Journal of Functional Analysis, 269(8):2427-2504, 2015.

[22] J. Földes, N. Glatt-Holtz, G. Richards, and J.P. Whitehead. Ergodicity in Randomly Forced Rayleigh-Bénard Convection. To appear in Nonlinearity, 2015. Available at http://arxiv.org/abs/1511.01247.

[23] N. E. Glatt-Holtz, D. P. Herzog, and J. C. Mattingly. Scaling and Saturation in Infinite-Dimensional Control Problems with Applications to Stochastic Partial Differential Equations. Annals of PDE, 2017. (to appear).

[24] D. Goluskin and E. A. Spiegel. Convection driven by internal heating. Physics Letters A, 377(1):83-92, 2012.

[25] M. Hairer and A. J. Majda. A simple framework to justify linear response theory. Nonlinearity, 23(4):909, 2010.

[26] M. Hairer and J. C. Mattingly. Ergodicity of the 2D Navier-Stokes equations with degenerate stochastic forcing. Ann. of Math. (2), 164(3):993-1032, 2006.

[27] M. Hairer and J. C. Mattingly. Spectral gaps in Wasserstein distances and the 2D stochastic Navier-Stokes equations. Ann. Probab., 36(6):2050-2091, 2008.

[28] M. Hairer and J. C. Mattingly. A Theory of Hypoellipticity and Unique Ergodicity for Semilinear Stochastic PDEs. Electron. J. Probab., 16(23):658-738, 2011.

[29] M. Hairer, J. C. Mattingly, and M. Scheutzow. Asymptotic coupling and a general form of Harris' theorem with applications to stochastic delay equations. Probab. Theory Related Fields, 149(1-2):223-259, 2011.

[30] R. Z. Khas'minskii. Ergodic properties of recurrent diffusion processes and stabilization of the solution to the Cauchy problem for parabolic equations. Theory of Probability Ėamp; Its Applications, 5(2):179-196, 1960.

[31] S. Kuksin and A. Shirikyan. Mathematics of Two-Dimensional Turbulence. Number 194 in Cambridge Tracts in Mathematics. Cambridge University Press, 2012.

[32] D. Lohse and K. Q. Xia. Small-scale properties of turbulent Rayleigh-Bénard convection. Annual Review of Fluid Mechanics, 42:335-364, 2010.

[33] L. Lu, C. Doering, and F. Busse. Bounds on convection driven by internal heating. Journal of mathematical physics, 45(7):2967-2986, 2004.

[34] P. Manneville. Rayleigh-Bénard Convection: Thirty Years of Experimental, Theoretical, and Modeling Work. In Dynamics of Spatio-Temporal Cellular Structures, pages 41-65. Springer, 2006.

[35] I. Nourdin and G. Peccati. Normal approximations with Malliavin calculus: from Stein's method to universality, volume 192. Cambridge University Press, 2012.

[36] D. Nualart. Malliavin calculus and its applications, volume 110 of CBMS Regional Conference Series in Mathematics. Published for the Conference Board of the Mathematical Sciences, Washington, DC, 2009.

[37] F. Otto and C. Seis. Rayleigh-Bénard convection: improved bounds on the Nusselt number. Journal of mathematical physics, 52(8):083702, 2011.

[38] J. Park. Dynamic bifurcation theory of Rayleigh-Bénard convection with infinite Prandtl number. Discrete Contin. Dyn. Syst., 6(3):591, 2006. 
[39] P. H. Roberts. Convection in horizontal layers with internal heat generation. Theory. Journal of Fluid Mechanics, 30(01):33-49, 1967.

[40] R. Temam. Navier-Stokes equations: Theory and numerical analysis. AMS Chelsea Publishing, Providence, RI, 2001. Reprint of the 1984 edition.

[41] D. J. Tritton and M. N. Zarraga. Convection in horizontal layers with internal heat generation. Experiments. Journal of Fluid Mechanics, 30(01):21-31, 1967.

[42] C. Villani. Optimal transport: old and new, volume 338. Springer Science \&amp; Business Media, 2008.

[43] X. Wang. Infinite Prandtl number limit of Rayleigh-Bénard convection. Comm. Pure Appl. Math., 57(10):1265-1282, 2004.

[44] X. Wang. Large Prandtl number behavior of the Boussinesq system of Rayleigh-Bénard convection. Appl. Math. Lett., 17(7):821-825, 2004.

[45] X. Wang. A note on long time behavior of solutions to the Boussinesq system at large Prandtl number. In Nonlinear partial differential equations and related analysis, volume 371 of Contemp. Math., pages 315-323. Amer. Math. Soc., Providence, RI, 2005.

[46] X. Wang. Asymptotic behavior of the global attractors to the Boussinesq system for Rayleigh-Bénard convection at large Prandtl number. Comm. Pure Appl. Math., 60(9):1293-1318, 2007.

[47] X. Wang. Bound on vertical heat transport at large Prandtl number. Phys. D, 237(6):854-858, 2008.

[48] X. Wang. Stationary statistical properties of Rayleigh-Bénard convection at large Prandtl number. Comm. Pure Appl. Math., 61(6):789-815, 2008.

[49] J. P. Whitehead and C. R. Doering. Internal heating driven convection at infinite Prandtl number. Journal of Mathematical Physics, 52(9):093101, 2011. 
Juraj Földes

Department of Mathematics

University of Virginia

Web: http://www.people.virginia.edu/ jf8dc/

Email: foldes@virginia.edu

Nathan Glatt-Holtz

Department of Mathematics

Tulane University

Web: http://www.math.tulane.edu/ negh/

Email: negh@tulane.edu
Geordie Richards

Department of Mechanical and Aerospace Engineering Utah State University

Web: www.geordierichards.com

Email: geordie.richards@usu.edu 